\title{
A coefficient inverse problem with a single measurement of phaseless scattering data
}

\author{
Michael V. Klibanov* Dinh-Liem Nguyen ${ }^{\dagger} \quad$ Loc H. Nguyen*
}

\begin{abstract}
This paper is concerned with a numerical method for a 3D coefficient inverse problem with phaseless scattering data. These are multi-frequency data generated by a single direction of the incident plane wave. Our numerical procedure consists of two stages. The first stage aims to reconstruct the (approximate) scattered field at the plane of measurements from its intensity. We present an algorithm for the reconstruction process and prove a uniqueness result of this reconstruction. After obtaining the approximate scattered field, we exploit a newly developed globally convergent numerical method to solve the coefficient inverse problem with the phased scattering data. The latter is the second stage of our algorithm. Numerical examples are presented to demonstrate the performance of our method. Finally, we present a numerical study which aims to show that, under a certain assumption, the solution of the scattering problem for the 3D scalar Helmholtz equation can be used to approximate the component of the electric field which was originally incident upon the medium.
\end{abstract}

Keywords. single measurement data, phaseless inverse scattering, uniqueness theorem, numerical method

AMS subject classification. 35R30, 78A46, 65C20

\section{Introduction}

The goal of this paper is to develop a new numerical method for a 3D phaseless coefficient inverse scattering problem in the case when the data to be inverted are generated by a single measurement event at multiple frequencies. We assume that only the intensity, i.e. the square modulus, of a complex valued wave field can be measured outside of scatterers and phase cannot be measured. We use only a single direction of the incident plane wave. In other words, we consider the phaseless coefficient inverse scattering problem with single measurement data. Thus, this is a non-overdetermined case, i.e. the number of free variables in the data equals to the number of free variables in the unknown coefficient. We propose a two-stage reconstruction procedure. In the first stage we approximately reconstruct the scattered wave field at the plane of measurements. Hence, this stage leads

${ }^{*}$ Department of Mathematics and Statistics, University of North Carolina at Charlotte, Charlotte, NC 28223; (mklibanv@uncc.edu, lnguye50@uncc.edu)

${ }^{\dagger}$ Department of Mathematics, Kansas State University, Manhattan, KS 66506 (dlnguyen@ksu.edu) 
to a conventional phased coefficient inverse scattering problem: when the whole complex valued wave field is known at a part of the measurement plane. Next, to reconstruct the unknown coefficient of Helmholtz equation, we apply the newly developed globally convergent algorithm of [32]. According to [8,32], we call a numerical method for a coefficient inverse problem globally convergent if a theorem is proven, which guarantees that this method delivers at least one point in a sufficiently small neighborhood of the exact solution without any a priori knowledge of this neighborhood.

Unlike the current paper, in [20] the case when the intensity is given on an interval of frequencies for multiple point sources was considered. While locations and shapes of unknown scatterers were imaged accurately in [20], the accuracy of reconstructed abnormality/background contrasts was poor. This is because a linearization of the travel time function was used in [20]. On the other hand, the globally convergent numerical inversion method of [32] provides very accurate locations and contrasts of abnormalities for a single measurement case. The latter was consistently demonstrated on both computationally simulated [32] and experimental data [33, 41] including the case when unknown targets were buried in a sandbox [40]. The arguments in this paragraph are the reasons of our choice of the two-stage procedure.

The study of the coefficient inverse problem with phaseless scattering data is motivated by applications in, e.g. imaging of nano-scale structures and biological cells. Typical nano structures of interest have sizes of hundreds of nanometers $(\mathrm{nm})$. Recall that 1 micron $(\mu m)=10^{3} \mathrm{~nm}$. Typical sizes of biological cells are in the range of $(5,100) \mu m[49,50]$. To image these, one should use optical sources with the same range of wavelengths. However, the corresponding frequency is very large. For example, the wavelength $\lambda=1 \mu \mathrm{m}$ corresponds to the frequency $\omega=299,792 \mathrm{GHz}$. Hence, only the intensity of the scattered field can be measured while the phase is lost $12,13,17,48,54$.

Solution of the coefficient inverse scattering problem without the phase information is a long standing problem. For the first time, this problem was probably posed in [10, Chapter 10]. The first uniqueness result for this problem was established in [21] in the 1D case, also see [1] for a follow up result. In 3D, the first uniqueness result was obtained in [22]. Later, uniqueness theorems in 3D were established in [23, 25, 31,57]. The analytic reconstruction procedures in $3 \mathrm{D}$ were proposed in $[26,27,29,30]$. However, these procedures require a large range of frequencies which might be unrealistic in practice. Hence, the method of 29] was modified and made suitable for computations for a realistic range of parameters in 20$]$.

In publications [44 46] phaseless coefficient inverse scattering problems were considered. Their statements are different from the ones in papers cited above. Uniqueness theorems were proved and reconstruction procedures were proposed in [44 46]. In [6, 7 a phaseless coefficient inverse scattering problem for Helmholtz equation was solved numerically using Kirchhoff migration and Born approximation. While coefficients of PDEs are subjects of interests in the above cited works, there is also a significant interest in the reconstruction of surfaces of scatterers from phaseless data. In this regard we refer to, e.g. publications $2,5,15,16,38,58$ and references cited therein.

Let $k>0$ be the wave number. In fact, on the first stage of our procedure we reconstruct the first term of the asymptotic expansion at $k \rightarrow \infty$ of the solution of Helmholtz equation at the measurement plane. We prove a theorem which claims that this reconstruction is unique. In this paper, for simplicity, we use the Helmholtz equation to model the light propagation. On the other hand, it is well-known that the wave field is 
governed by the Maxwell's system. To validate our analysis, we numerically compare in Appendix the solution of the Helmholtz equation with the one of the Maxwell's system. Another validation can be attributed to quite accurate results obtained by this group for phased microwave experimental data [33,40,41] in which the Helmholtz equation was used and the globally convergent numerical method of [32] was applied.

In the next section, we formulate the phaseless coefficient inverse scattering problem. In Section 3, we prove a uniqueness result of the phase retrieval. In Section 4, we describe our numerical approach to reconstruct the lost phase. In Section 5, we briefly summarize our globally convergent method of [32] for the reader's convenience. In Section 6, we present our numerical results. In Section 7 we provide summary of our results. Finally, in the Appendix, which is Section 8, we compare numerically solutions to Maxwell's system and Helmholtz equation.

\section{Problem Statement}

Let a laser beam illuminate the unknown nano-structure/biological cell, which plays the role of a scatterer. The diameter of a laser beam is a few millimeters $(\mathrm{mm})$ and $1 \mathrm{~mm}=$ $10^{3} \mu \mathrm{m}$. Given that sizes of our scatterers do not exceed $100 \mu \mathrm{m}=0.1 \mathrm{~mm}$ (section 1 ), these scatterers "percept" that laser beam as a perfect plane wave. The laser beam scatters after hitting the scatterers. It is well known that modern light detectors placed inside of the laser beam are burned. Hence, one should place detectors outside of that beam. However, outside of the laser beam the total wave field approximately equals the scattered wave field. Hence, we assume below that we measure the intensity of the scattered wave at a square $P_{\text {meas }}$ of a fixed plane, see Figure 1 for an illustration. The problem we consider in this paper is to reconstruct the spatially distributed dielectric constant of the scatterer from the measurements of the intensities of the scattered waves on $P_{\text {meas }}$ at an interval of frequencies. We point out, however, that a precise mathematical modeling of the laser beam is outside of the scope of this publication. So, the above considerations were given only to explain why do we consider the intensity of the scattered rather than of the total wave field.

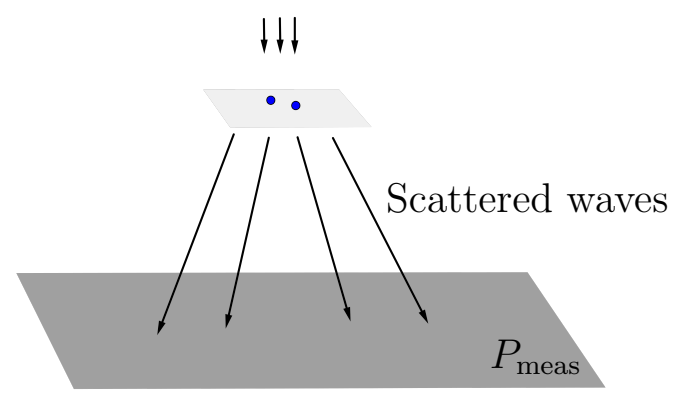

Figure 1: The experimental setup. The laser beam hits scatterer (dots) and causes scattered wave. The intensity is measured on $P_{\text {meas }}$. 


\subsection{The phaseless coefficient inverse scattering problem}

Denote $\mathbf{x}=\left(x_{1}, x_{2}, x_{3}\right) \in \mathbb{R}^{3}$. Let $\Omega$ be a bounded domain in $\mathbb{R}^{3}$ with a smooth boundary $\partial \Omega$ and such that $\Omega \subset\left\{x_{3}>0\right\}$. Let $c(\mathbf{x})$, be a function satisfying the following conditions:

$$
c(\mathbf{x}) \in C^{15}\left(\mathbb{R}^{3}\right), \quad c(\mathbf{x}) \geq 1 \text { for all } \mathbf{x} \in \mathbb{R}^{3} \quad \text { and } c(\mathbf{x})=1 \text { for all } \mathbf{x} \in \mathbb{R}^{3} \backslash \Omega .
$$

The function $c(\mathbf{x})$ models the spatially distributed dielectric constant of the medium consisting of the background and the scatterers. The condition $c(\mathbf{x})=1$ in $\mathbb{R}^{3} \backslash \Omega$ means that we suitably scale the dielectric constant, so that it equals to 1 in the background (vacuum). The condition $c(\mathbf{x}) \geq 1$ means that the dielectric constant in the medium is not less than in the vacuum. The Riemannian metric corresponding to the function $c(\mathbf{x})$ is given by

$$
d \tau(\mathbf{x})=\sqrt{c(\mathbf{x})}|d \mathbf{x}|, \quad|d \mathbf{x}|=\sqrt{\left(d x_{1}\right)^{2}+\left(d x_{2}\right)^{2}+\left(d x_{3}\right)^{2}} .
$$

Fix the number $a>0$. Consider the plane $P_{a}=\left\{\left(x_{1}, x_{2},-a\right): x_{1}, x_{2} \in \mathbb{R}\right\}$.We impose the following condition on the function $c(\mathbf{x})$ :

Assumption 2.1 (Assumption of Regularity of Geodesic Lines.). For any point $\mathbf{x} \in \mathbb{R}^{3}$ there exists a unique geodesic line $\Gamma(\mathbf{x}, a)$, with respect to the metric $d \tau$, connecting $\mathbf{x}$ with the plane $P_{a}$ and perpendicular to $P_{a}$.

The following sufficient condition of the regularity of geodesic lines was derived in 53

$$
\sum_{i, j=1}^{3} \frac{\partial^{2} c(\mathbf{x})}{\partial x_{i} \partial x_{j}} \xi_{i} \xi_{j} \geq 0, \forall \mathbf{x} \in \bar{\Omega}, \forall \boldsymbol{\xi} \in \mathbb{R}^{3} .
$$

Remark 2.1. The smoothness condition (2.1) imposed on the function $c(\mathbf{x})$ as well as Assumption 2.1 are necessary for the theoretical purposes only: to derive the asymptotic behavior (3.1). However, we do not verify neither condition (2.1) nor Assumption 2.1 in our numerical studies. Indeed, that asymptotic behavior is derived in Theorem 3.1 on the basis of the construction of the solution of the Cauchy problem for a hyperbolic equation. However, this construction requires that $c(\mathbf{x}) \in C^{15}\left(\mathbb{R}^{3}\right)[30,52]$. Besides, the minimal smoothness of unknown coefficients is usually not of a great concern in studies of coefficient inverse problems, see, e.g. [42.43] and theorem 4.1 in [51].

The function $\tau(\mathbf{x})$ is the travel time from the plane $P_{a}$ to the point $\mathbf{x}$ and [30]

$$
\tau(\mathbf{x})=\int_{\Gamma(\mathbf{x}, a)} \sqrt{c(\boldsymbol{\xi})} d \sigma .
$$

Lemma 2.1. For all $\mathbf{x} \in\left\{x_{3}>-a\right\}$ we have $\tau(\mathbf{x}) \geq x_{3}$. Consider the set $\Omega_{1}$,

$$
\Omega_{1}=\{\mathbf{x}: c(\mathbf{x})>1\} \subset \Omega .
$$

Assume that the set (2.3) is convex and its boundary $\partial \Omega_{1} \in C^{1}$. Then $\tau(\mathbf{x})=x_{3}$ if and only if $\Gamma(\mathbf{x}, a)=L(\mathbf{x}, a)$, where $L(\mathbf{x}, a)$ is the straight line connecting the point $\mathbf{x}$ with the plane $P_{a}$ and orthogonal to $P_{a}$. If $\tau(\mathbf{x})=x_{3}$, then for any point $\mathbf{x}^{\prime} \in \Gamma(\mathbf{x}, a)=L(\mathbf{x}, a)$, except of probably one point, there exists such a sufficiently small neighborhood $O\left(\mathbf{x}^{\prime}\right)$ of $\mathbf{x}^{\prime}$ that $\tau\left(\mathbf{x}^{\prime \prime}\right)=x_{3}^{\prime \prime}, \forall \mathbf{x}^{\prime \prime}=\left(x_{1}^{\prime \prime}, x_{2}^{\prime \prime}, x_{3}^{\prime \prime}\right) \in O\left(\mathbf{x}^{\prime}\right)$. 
Proof. The inequality $\tau(\mathbf{x}) \geq x_{3}$ follows from (2.2) as well as from the fact that by (2.1) $c(\mathbf{x}) \geq 1$. Suppose now that $\tau(\mathbf{x})=x_{3}$. If $\Gamma(\mathbf{x}, a) \cap \Omega_{1} \neq \varnothing$, then $(2.2)$ implies that $\tau(\mathbf{x})>x_{3}$. Hence, $\Gamma(\mathbf{x}, a) \cap \Omega_{1}=\varnothing$. This means that $\Gamma(\mathbf{x}, a)=L(\mathbf{x}, a)$. Now, either $L(\mathbf{x}, a) \cap \partial \Omega_{1}=\varnothing$ or, due to both the convexity of the domain $\Omega_{1}$ and the smoothness of its boundary $\partial \Omega_{1}$, the straight line $L(\mathbf{x}, a)$ is the tangent line to $\partial \Omega_{1}$ at a certain unique point. Obviously in both these two cases the assertion of this lemma about $O\left(\mathbf{x}^{\prime}\right)$ is true. $\square$ Let $[\underline{k}, \bar{k}]$ be an interval of wave numbers $k=2 \pi / \lambda \in[\underline{k}, \bar{k}]$ where $\lambda$ is the dimensionless wavelength. Consider the incident plane wave $u_{\text {inc }}(\mathbf{x}, k)$ propagating along the $x_{3}$ axis,

$$
u_{\text {inc }}(\mathbf{x}, k)=\exp \left(\mathrm{i} k x_{3}\right) \text {. }
$$

The propagation of the total wave field $u(\mathbf{x}, k)$ is governed by the Helmholtz equation and the outgoing Sommerfeld radiation condition,

$$
\begin{gathered}
u(\mathbf{x}, k)=u_{\text {inc }}(\mathbf{x}, k)+u_{\mathrm{sc}}(\mathbf{x}, k), \quad \mathbf{x} \in \mathbb{R}^{3}, k \in[\underline{k}, \bar{k}], \\
\left\{\begin{array}{cl}
\Delta u(\mathbf{x}, k)+k^{2} c(\mathbf{x}) u(\mathbf{x}, k)=0, & \mathbf{x} \in \mathbb{R}^{3}, \\
\partial_{r} u_{\mathrm{sc}}(\mathbf{x}, k)-\mathrm{i} k u_{\mathrm{sc}}(\mathbf{x}, k)=o\left(r^{-1}\right), & \text { as } r=|\mathbf{x}| \rightarrow \infty .
\end{array}\right.
\end{gathered}
$$

Let the number $R>0$. Denote

$$
P_{\text {meas }}=\left\{\mathbf{x}=\left(x_{1}, x_{2}, x_{3}\right):-b<x_{1}, x_{2}<b, x_{3}=R\right\}
$$

the square on the plane $P=\left\{x_{3}=R\right\}$ where measurements of the intensity $|u(\mathbf{x}, k)|^{2}$ are conducted. Here $R>0$ is the distance from the origin to the measurement plane and $b>0$ is the size of that rectangle. Assume that the plane $P$ does not intersect with $\Omega$, $\bar{\Omega} \cap P=\varnothing$. The phaseless coefficient inverse scattering problem is formulated as:

Phaseless Coefficient Inverse Scattering Problem (PCISP). Given the data

$$
f(\mathbf{x}, k)=\left|u_{\mathrm{sc}}(\mathbf{x}, k)\right|^{2}, \quad \mathbf{x} \in P_{\text {meas }}, k \in[\underline{k}, \bar{k}],
$$

determine the dielectric constant $c(\mathbf{x})$ for $\mathbf{x} \in \Omega$.

Remark 2.2. $\quad$ 1. We model the wave propagation by the single Helmholtz equation with the outgoing radiation condition instead of the full Maxwell's system, see section 9 and a discussion in Introduction.

2. A natural question about the uniqueness of the PCISP arises. We prove in Section 3 that one can uniquely reconstruct the first term of the asymptotic expansion at $k \rightarrow \infty$ of the function $u(\mathbf{x}, k), \mathbf{x} \in P_{\text {meas }}$ from the data (2.8). Let the square $P_{\text {meas }} \subset P$, where $P$ is the corresponding plane. The next question, however, is about the uniqueness of the reconstruction of the coefficient $c(\mathbf{x})$ for $\mathbf{x} \in \Omega$ even in the case when the whole function $u(\mathbf{x}, k)$ (rather than that first term only) is known for all $\mathbf{x} \in P$ and for all $k>0$. Addressing this question is a well known long standing open problem. Indeed, all uniqueness theorems for $n-D, n \geq 2$ coefficient inverse problems with single measurement data are currently proven only by the method, which was originally proposed in [9] in 1981, also see, e.g. the section 1.10 in the book [8], the book [18], the survey [19] and references cited in [19]. Carleman estimates are the key ingredient of this method. However, in our specific case, this method works only if the right hand side of Helmholtz equation (2.6) is non vanishing in $\bar{\Omega}$. Hence, we just assume uniqueness of that second problem: for the purpose of computations. 


\subsection{The Lippman-Schwinger equation}

Assume that the function $c(\mathbf{x})$ satisfying $(2.1)$ is known for all $\mathbf{x} \in \mathbb{R}^{3}$. It follows from the Helmholtz equation in (2.6) that

$$
\begin{gathered}
\Delta u_{\mathrm{sc}}(\mathbf{x}, k)+k^{2} u_{\mathrm{sc}}(\mathbf{x}, k)+k^{2} \beta(\mathbf{x}) u(\mathbf{x}, k)=0, \forall \mathbf{x} \in \mathbb{R}^{3}, \\
\beta(\mathbf{x})=c(\mathbf{x})-1 .
\end{gathered}
$$

Here, we have used (2.5) and the fact that $\Delta u_{0}(\mathbf{x}, k)+k^{2} u_{0}(\mathbf{x}, k)=0$. This and the outgoing Sommerfeld radiation condition in (2.6) imply that

$$
u_{\mathrm{sc}}(\mathbf{x}, k)=k^{2} \int_{\mathbb{R}^{3}} \frac{\exp (\mathrm{i} k|\mathbf{x}-\boldsymbol{\xi}|)}{4 \pi|\mathbf{x}-\boldsymbol{\xi}|} \beta(\mathbf{x}) u(\boldsymbol{\xi}, k) d \boldsymbol{\xi} .
$$

Using (2.5) and (2.10), we obtain the Lippmann-Schwinger equation

$$
u(\mathbf{x}, k)=u_{\text {inc }}(\mathbf{x}, k)+k^{2} \int_{\Omega} \frac{\exp (\mathrm{i} k|\mathbf{x}-\boldsymbol{\xi}|)}{4 \pi|\mathbf{x}-\boldsymbol{\xi}|} \beta(\boldsymbol{\xi}) u(\boldsymbol{\xi}, k) d \boldsymbol{\xi} .
$$

For an integer $k \geq 0$ and for $\alpha \in(0,1)$ let $C^{k+\alpha}\left(\mathbb{R}^{3}\right)$ be Hölder spaces. The following result holds [11, Chapter 8]:

Theorem 2.1. Assume that $c(\mathbf{x}) \in C^{\alpha}\left(\mathbb{R}^{3}\right)$ satisfies the rest of conditions (2.1). Then the Lippmann-Schwinger equation (2.11) has unique solution $u(\mathbf{x}, k) \in C^{2+\alpha}\left(\mathbb{R}^{3}\right)$ for all $k>0$. Moreover, this function $u(\mathbf{x}, k)$ is the unique solution of the problem (2.6).

When using the globally convergent method [32] below, we solve equation (2.11) on each iteration. To solve integral equation (2.11) numerically, we use the method developed in 35,39 .

\section{Uniqueness result}

In this section, we prove that the first term of the asymptotic expansion of the function $u(\mathbf{x}, k)$ at $k \rightarrow \infty$ for points $\mathbf{x} \in P_{\text {meas }}$ can be determined uniquely from the data $f(\mathbf{x}, k)$. For any number $\theta>0$ we define the half-plane $\mathbb{C}_{\theta}$ of the complex plane $\mathbb{C}$ as

$$
\mathbb{C}_{\theta}=\{z \in \mathbb{C}: \Im z>-\theta\}
$$

Theorem 3.1. Assume that the function c $(\mathbf{x})$ satisfies conditions (2.1). Suppose that the Assumption of the Regularity of Geodesic Lines holds true. Let $G \subset\left\{\mathbf{x}=\left(x_{1}, x_{2}, x_{3}\right)\right.$ : $\left.x_{3}>-a\right\}$ be an arbitrary bounded domain such that $P_{\text {meas }} \subset G$. Then there exists a number $\theta=\theta(G)>0$ such that for all points $\mathbf{x} \in G$, the solution $u(\mathbf{x}, k)$ of problem (2.5) - (2.6) is analytic with respect to $k \in \mathbb{R}$ and can be analytically continued in the half-plane $\mathbb{C}_{\theta}$. Furthermore, the function $f(\mathbf{x}, k)=\left|u_{s c}(\mathbf{x}, k)\right|^{2}, \mathbf{x} \in P_{\text {meas }}$ is analytic with respect to $k \in \mathbb{R}$. In addition, the following asymptotic behavior holds

$$
u_{\mathrm{sc}}(\mathbf{x}, k)=A(\mathbf{x}) e^{\mathrm{i} k \tau(\mathbf{x})}-e^{\mathrm{i} k x_{3}}+\mu(\mathbf{x}, k), \quad \mathbf{x} \in G, k \rightarrow \infty
$$


where the function $A(\mathbf{x})>0$, the function $\tau(\mathbf{x})$ is defined in Subsection 2.1, and the function $\mu(\mathbf{x}, k)$ is such that for $j=0,1,2$

$$
\partial_{k}^{j} \mu(\mathbf{x}, k)=O\left(k^{-1}\right), \quad \mathbf{x} \in G, k \rightarrow \infty .
$$

In the proof of this theorem, we modify the material of section 4 of [30]. Although properties (3.1), (3.2) and the analyticity of the function $u(\mathbf{x}, k)$ follow from results of [30], they are not explicitly formulated there.

Proof of Theorem 3.1. Consider the following auxiliary hyperbolic equation

$$
c(\mathbf{x}) v_{t t}=\Delta v, \quad \mathbf{x} \in \mathbb{R}^{3}, t \in \mathbb{R} .
$$

And consider the solution of equation (3.1) in the form

$$
v(\mathbf{x}, t)=\delta\left(t-x_{3}\right)+\widetilde{v}(\mathbf{x}, t)
$$

where $\widetilde{v}(\mathbf{x}, t)$ is such that

$$
\widetilde{v}(\mathbf{x}, t)=0 \quad \text { for } x_{3}<-a .
$$

Let $T>0$ be an arbitrary number. Denote

$$
\begin{gathered}
D(T)=\{(\mathbf{x}, t): \max (-a, \tau(\mathbf{x}))<t<T\}, \\
H(t)= \begin{cases}1 & t>0, \\
0 & t<0 .\end{cases}
\end{gathered}
$$

It was proven in [30, Theorem 1] that the problem $(3.3)-(3.5)$ has unique solution of the form

$$
v(\mathbf{x}, t)=A(\mathbf{x}) \delta(t-\tau(\mathbf{x}))+H(t-\tau(\mathbf{x})) \widehat{v}(\mathbf{x}, t),
$$

where the function $A(\mathbf{x})>0$ and

$$
\widehat{v}(\mathbf{x}, t) \in C^{2}(\overline{D(T)}) .
$$

Furthermore, Theorem 4 of Chapter 10 of [56] as well as Remark 3 after that theorem guarantee that there exists a number $\theta=\theta(c, G)>0$ and a number $C_{1}=C_{1}(c, G)>$ $0, C_{2}=C_{2}(c, G)>0$, all three depending only on listed parameters, such that

$$
\left|D_{\mathbf{x}}^{\alpha} D_{t}^{k} \widehat{v}(\mathbf{x}, t)\right| \leq C_{2} e^{-\theta t}, \quad \forall \mathbf{x} \in G, \forall t>C_{1},|\alpha|+k \leq 2 .
$$

Here $\alpha=\left(\alpha_{1}, \alpha_{2}, \alpha_{3}\right)$ is the multiindex with non-negative integer coordinates and $|\alpha|=$ $\alpha_{1}+\alpha_{2}+\alpha_{3}$. By (3.6) and (3.8) we can consider Fourier transform of the function $v(\mathbf{x}, t)$,

$$
V(\mathbf{x}, k)=\int_{-\infty}^{\infty} v(\mathbf{x}, t) e^{\mathrm{i} k t} d t=A(\mathbf{x}) e^{\mathrm{i} k \tau(\mathbf{x})}+\int_{\tau(\mathbf{x})}^{\infty} \widehat{v}(\mathbf{x}, t) e^{\mathrm{i} k t} d t .
$$

Next, Theorem 3.3 of [55] and Theorem 6 of Chapter 9 of [56] imply that

$$
V(\mathbf{x}, k)=u(\mathbf{x}, k), \quad \forall \mathbf{x} \in \mathbb{R}^{3}, \forall k>0,
$$


where $u(\mathbf{x}, k)$ is the solution of our original forward problem (2.5)-(2.6). Next, using (3.6)-3.8), 3.10) and the integration by parts in (3.9), we obtain

$$
u(\mathbf{x}, k)=A(\mathbf{x}) e^{\mathrm{i} k \tau(\mathbf{x})}+\frac{\mathrm{i}}{k} \widehat{v}(\mathbf{x}, \tau(\mathbf{x})) e^{\mathrm{i} k \tau(\mathbf{x})}+\frac{\mathrm{i}}{k} \int_{\tau(\mathbf{x})}^{\infty} \partial_{t} \widehat{v}(\mathbf{x}, t) e^{\mathrm{i} k t} d t, \quad k>0,
$$

which proves the asymptotic expansion (3.1), 3.2).

Next, it follows from $3.6-3.10$ that

$$
\partial_{k} V(\mathbf{x}, k)=\mathrm{i} A(\mathbf{x}) \tau(\mathbf{x}) e^{\mathrm{i} k \tau(\mathbf{x})}+\mathrm{i} \int_{\tau(\mathbf{x})}^{\infty} \widehat{v}(\mathbf{x}, t) t e^{\mathrm{i} k t} d t, \quad \forall k \in \mathbb{C}_{\theta} .
$$

Hence, using (3.10), we conclude that the function $u(\mathbf{x}, k)$ has analytic continuation with respect to $k$ from the real line $\mathbb{R}$ in the half-plane $\mathbb{C}_{\theta}$. Finally, the analyticity of the function $f(\mathbf{x}, k)=\left|u_{\mathrm{sc}}(\mathbf{x}, k)\right|^{2}$ with respect to $k \in \mathbb{R}$ follows from [25, Lemma 3.5].

Theorem 3.2 is similar with theorem 1 of [31, Lemma 3.5]. While theorem 1 of [31, Lemma 3.5] works for the case when the wave field is generated by a point source, Theorem 3.2 is valid for the case of the incident plane wave. The proof here is different from the one in [31, Lemma 3.5]. Two major differences are that neither an analog of Lemma 2.1 nor the derivative $\varphi^{\prime}(k)$ in (3.17) were not considered in [31, Lemma 3.5].

Theorem 3.2. Assume that the function c $(\mathbf{x})$ satisfies condition (2.1). Suppose that the Assumption of the Regularity of Geodesic Lines holds true. In addition, assume that conditions of Lemma 2.1 hold. Consider an arbitrary point $\mathbf{x}_{0} \in P_{\text {meas }}$. Also, consider the function $\varphi(k)$,

$$
\varphi(k)=\left|u_{s c}\left(\mathbf{x}_{0}, k\right)\right|^{2}, k \in(\underline{k}, \bar{k}),
$$

where $(\underline{k}, \bar{k}) \subset \mathbb{R}$ is a certain interval. Then the numbers $\tau\left(\mathbf{x}_{0}\right)$ and $A\left(\mathbf{x}_{0}\right)$ in the asymptotic expansion (3.1), (3.2) are uniquely determined from the knowledge of the function $\varphi(k)$ in (3.11). Furthermore, if $\tau\left(\mathbf{x}_{0}\right)=x_{3,0}$ for $\mathbf{x}_{0}=\left(x_{1,0}, x_{2,0}, x_{3,0}\right)$, then $A\left(\mathbf{x}_{0}\right)=1$.

Corollary 3.1 follows immediately from Theorem 3.2 .

Corollary 3.1. Consider the PCISP. Then functions $A(\mathbf{x})$ and $\tau(\mathbf{x})$ in the asymptotic expansion (3.1), (3.2) are uniquely determined for $\mathbf{x} \in P_{\text {meas }}$ from the knowledge of the function $f(\mathbf{x}, k)$ in $(2.8)$.

Proof of Theorem 3.2. For brevity denote $A:=A\left(\mathbf{x}_{0}\right), \tau:=\tau\left(\mathbf{x}_{0}\right)$. Since by Theorem 3.1. the function $\varphi(k)$ is analytic for $k \in \mathbb{R}$, then we assume below in this proof that the function $\varphi(k)$ is given for all $k \in \mathbb{R}$. Using (3.1), (3.2) and (3.11), we obtain for sufficiently large $k$

$$
\varphi(k)=A^{2}-2 A \cos \left[k\left(\tau-x_{3}\right)\right]+p(k),
$$

where the real valued function $p(k)$ is such that

$$
p^{(j)}(k)=O\left(k^{-1}\right), \quad k \rightarrow \infty, j=0,1,2 .
$$


It follows from 3.12 and 3.13 that $\lim _{k \rightarrow \infty} \varphi(k)$ exists if and only if $\tau=x_{3}$. Hence, assume first that $\tau=x_{3}$. To find the number $A$, we use the formula (4.16) of [30, Theorem $1]$,

$$
A\left(\mathbf{x}_{0}\right)=\exp \left(-\frac{1}{2} \int_{\Gamma\left(\mathbf{x}_{0}, a\right)} \frac{1}{c(\boldsymbol{\xi})} \Delta_{\xi} \tau(\boldsymbol{\xi}) d \sigma\right) .
$$

By Lemma 2.1 $\Gamma\left(\mathbf{x}_{0}, a\right)=L\left(\mathbf{x}_{0}, a\right)$. Furthermore, it follows from the assertion of that lemma about $O\left(\mathbf{x}^{\prime}\right)$ that $\Delta_{\xi} \tau(\boldsymbol{\xi})=0$ for all points $\boldsymbol{\xi} \in \Gamma\left(\mathbf{x}_{0}, a\right)$, except of probably one point. Hence, (3.14) implies that $A\left(\mathbf{x}_{0}\right)=1$.

Consider now the case $\tau \neq x_{3}$. Denote

$$
\alpha\left(\mathbf{x}_{0}\right)=\alpha=\tau\left(\mathbf{x}_{0}\right)-x_{3} .
$$

Lemma 2.1 implies that

$$
\alpha>0 .
$$

By 3.12 and 3.13

$$
\varphi^{\prime}(k)=2 A \alpha \sin (k \alpha)+p^{\prime}(k) .
$$

For sufficiently large $k$, consider the equation

$$
\varphi^{\prime}(k)=0 .
$$

Consider the real valued function $q(k)$,

$$
q(k)=-\frac{p^{\prime}(k)}{2 A \alpha} .
$$

Then, using (3.13)-(3.17), we obtain that equation (3.18) is equivalent with

$$
\begin{gathered}
\sin (k \alpha)=q(k), \\
q(k)=O\left(\frac{1}{k}\right), q^{\prime}(k)=O\left(\frac{1}{k}\right), k \rightarrow \infty .
\end{gathered}
$$

Consider a sufficiently large integer $n>1$. By (3.16) we can choose a sufficiently large $k>0$ such that

$$
k \alpha \in((n-1) \pi, n \pi+1)
$$

Since by 3.20$)|q(k)|<1$ for sufficiently large $k>0$, then it follows from (3.19)-(3.21) that

$$
k \alpha=(-1)^{n} \arcsin (q(k))+n \pi .
$$

We show now that equation 3.22 has unique solution

$$
k \in\left(\frac{(n-1) \pi}{\alpha}, \frac{n \pi+1}{\alpha}\right)=I_{n} .
$$

Indeed, consider the function $h_{n}(k)$,

$$
h_{n}(k)=k \alpha-(-1)^{n} \arcsin (q(k))-n \pi, k \in I_{n} .
$$


By (3.20) we can assume that

$$
|\arcsin (q(k))|<\frac{1}{2}, k \in I_{n} .
$$

Using (3.23) and (3.24), we obtain

$$
\begin{gathered}
h_{n}\left(\frac{(n-1) \pi}{\alpha}\right)=-\pi-(-1)^{n} \arcsin \left(q\left(\frac{(n-1) \pi}{\alpha}\right)\right)<-2<0, \\
h_{n}\left(\frac{n \pi+1}{\alpha}\right)=1-(-1)^{n} \arcsin \left(q\left(\frac{n \pi+1}{\alpha}\right)\right)>\frac{1}{2}>0 .
\end{gathered}
$$

It follows from (3.25) and (3.26) that the function $h_{n}(k)$ has at least one zero inside of the interval $I_{n}$. To show that this zero is unique, consider the derivative $h_{n}^{\prime}(k)$,

$$
h_{n}^{\prime}(k)=\alpha-(-1)^{n} \frac{q^{\prime}(k)}{\sqrt{1-q^{2}(k)}}, k \in I_{n} .
$$

Since $n>1$ is sufficiently large and $k \in I_{n}$, we can assume by 3.20 that

$$
\left|\frac{q^{\prime}(k)}{\sqrt{1-q^{2}(k)}}\right|<\frac{\alpha}{2} \text {. }
$$

Hence, $h_{n}^{\prime}(k)>\alpha / 2>0$ for $k \in I_{n}$. Hence, the function $h_{n}(k)$ is monotonically increasing on the interval $I_{n}$. Hence, the above mentioned zero of the function $h_{n}(k)$ on the interval $I_{n}$ is unique. We denote this zero $k_{n}$.

By $3.20-(3.22)$

$$
\begin{gathered}
k_{n} \alpha=n \pi+O\left(\frac{1}{n}\right), n \rightarrow \infty, \\
k_{n+1} \alpha=(n+1) \pi+O\left(\frac{1}{n}\right), n \rightarrow \infty .
\end{gathered}
$$

Hence,

$$
\left(k_{n+1}-k_{n}\right) \alpha=\pi+O\left(\frac{1}{n}\right), n \rightarrow \infty .
$$

In particular, it follows from (3.27) that $k_{n+1}-k_{n} \geq \pi / 2 \neq 0$ for sufficiently large $n$. Thus, we obtain from 3.27

$$
\alpha=\lim _{n \rightarrow \infty} \frac{\pi}{k_{n+1}-k_{n}} .
$$

Since the function $\varphi^{\prime}(k)$ is known, then all its zeros are also known. Next, since equations (3.18) and (3.19) are equivalent, then (3.28) uniquely defines the number $\tau=\alpha+x_{3}$ as

$$
\tau=x_{3}+\lim _{n \rightarrow \infty} \frac{\pi}{k_{n+1}-k_{n}} .
$$

Finally (3.13) and 3.17) imply that

$$
A=\frac{1}{2 \alpha} \lim _{n \rightarrow \infty} \varphi^{\prime}\left[\frac{1}{\alpha}\left(\frac{\pi}{2}+2 n \pi\right)\right] .
$$

The proof is complete. 


\section{Numerical method for the approximate phase re- trieval for $\mathrm{x} \in P_{\text {meas }}$}

In this section, we show how to recover functions $\tau(\mathbf{x})$ and $A(\mathbf{x})$ for $\mathbf{x} \in P_{\text {meas }}$ from the function $f(\mathbf{x}, k)$ in 2.8$)$. Everywhere in this section $\mathbf{x} \in P_{\text {meas }}$. By Theorem 3.1

$$
u(\mathbf{x}, k)=A(\mathbf{x}) \exp (\mathrm{i} k \tau(\mathbf{x}))(1+O(1 / k)), k \rightarrow \infty,
$$

where the function $u(\mathbf{x}, k)$ is the solution of $(2.6)$. In particular, this means that $|u(\mathbf{x}, k)| \approx$ $A(\mathbf{x})$ for sufficiently large $k$. We show below in this section how to approximate the functions $A(\mathbf{x})$ and $\tau(\mathbf{x})$ from the data $f(\mathbf{x}, k), \mathbf{x} \in P_{\text {meas }}, k \in[\underline{k}, \bar{k}]$. Dropping the remainder term $O(1 / k)$ in (4.1), we deduce from (2.5) that

$$
u_{\mathrm{sc}}(\mathbf{x}, k)=A(\mathbf{x}) \exp (\mathrm{i} k \tau(\mathbf{x}))-\exp \left(\mathrm{i} k x_{3}\right) .
$$

Hence, for $\alpha(\mathbf{x})$ defined in 3.15 , the data is approximated as

$$
f(\mathbf{x}, k)=\left|u_{\mathrm{sc}}(\mathbf{x}, k)\right|^{2}=A^{2}(\mathbf{x})+1-2 A(\mathbf{x}) \cos (k \alpha(\mathbf{x})) .
$$

Remark 4.1 ( 29,30$])$. Fix $\mathbf{x}$ in $P_{\text {meas }}$. One can approximate $A(\mathbf{x})$ and $\alpha(\mathbf{x})$ by calculating the period of the function $k \mapsto f(\mathbf{x}, k)$. For instance, we can find two consecutive local minimizers (or maximizers) $\kappa_{1}$ and $\kappa_{2}$ of $f(\mathbf{x}, k)$ in $[\underline{k}, \bar{k}]$. Thus, $\cos \left(\kappa_{1} \alpha(\mathbf{x})\right)=$ $\cos \left(\kappa_{2} \alpha(\mathbf{x})\right)=1$ and

$$
\alpha(\mathbf{x})=\frac{2 \pi}{\left|\kappa_{2}-\kappa_{1}\right|}
$$

This method can be used in theory. However, in some physical situations, the interval $[\underline{k}, \bar{k}]$ is not large enough for us to find two local minima of $f(\mathbf{x}, k)$. In addition, we might have errors when finding local minimizers due to the noise added to the data, see Figure 2 for an illustration.

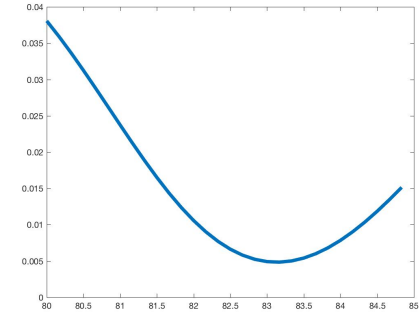

(a) Noiseless data.

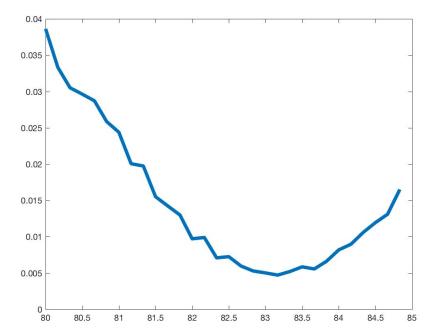

(b) Data with $10 \%$ noise

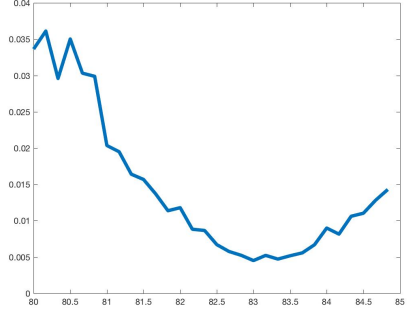

(c) Data with $15 \%$ noise

Figure 2: A typical example for the data $f(\mathbf{x}, k)$ when $k$ varies in $[\underline{k}, \bar{k}]=[80,85]$ where $[80,85]$ is a realistic range of wave numbers, see Section 6.3. In (a), the data attains only one minimum value, which does not provide enough data to apply (4.4). The reconstruction is even more inconvenient when the data, with noise, attains multiple extrema in the case (b) and (c).

Modifying the phase reconstruction procedure of [20] where the incident wave is a point source rather than the plane wave of our case, we propose the following reconstruction 
process. Fix a point $\mathbf{x} \in P_{\text {meas }}$ and let $k \in[\underline{k}, \bar{k}]$. Assuming that $\alpha(\mathbf{x}) \neq 0$, introduce $F_{1}(\mathbf{x}, k)$ as

$$
\begin{aligned}
F_{1}(\mathbf{x}, k) & =\int_{\underline{k}}^{k} f(\mathbf{x}, \kappa) d \kappa=\left(A^{2}(\mathbf{x})+1\right)(k-\underline{k})-\frac{2 A(\mathbf{x})}{\alpha(\mathbf{x})}(\sin (k \alpha(\mathbf{x}))-\sin (\underline{k} \alpha(\mathbf{x}))) \\
& =\left(A^{2}(\mathbf{x})+1\right)(k-\underline{k})+\frac{2 A(\mathbf{x})}{\alpha(\mathbf{x})} \sin (\underline{k} \alpha(\mathbf{x}))-\frac{2 A(\mathbf{x})}{\alpha(\mathbf{x})} \sin (k \alpha(\mathbf{x})) .
\end{aligned}
$$

Next, we define

$$
\begin{aligned}
F_{2}(\mathbf{x}, k)= & \int_{\underline{k}}^{k} F_{1}(\mathbf{x}, \kappa) d \kappa=\frac{\left(A^{2}(\mathbf{x})+1\right)}{2}(k-\underline{k})^{2} \\
& +\frac{2 A(\mathbf{x}) \sin (\underline{k} \alpha(\mathbf{x}))}{\alpha(\mathbf{x})}(k-\underline{k})+\frac{2 A(\mathbf{x})}{\alpha^{2}(x)} \cos (k \alpha(x))-\frac{2 A(\mathbf{x})}{\alpha^{2}(x)} \cos (\underline{k} \alpha(x)) .
\end{aligned}
$$

Combining (4.3) and 4.5 gives

$$
\begin{aligned}
& \alpha^{2}(\mathbf{x}) F_{2}(\mathbf{x}, k)=\frac{\alpha^{2}(\mathbf{x})\left(A^{2}(\mathbf{x})+1\right)}{2}(k-\underline{k})^{2} \\
& \quad+2 \alpha(\mathbf{x}) A(\mathbf{x}) \sin (\underline{k} \alpha(\mathbf{x}))(k-\underline{k})+A^{2}(\mathbf{x})+1-f(\mathbf{x}, k)-2 A(\mathbf{x}) \cos (\underline{k} \alpha(x)) .
\end{aligned}
$$

Equation (4.6) can be rewritten as

$$
F_{2}(\mathbf{x}, k) \xi_{1}(\mathbf{x})+(k-\underline{k})^{2} \xi_{2}(\mathbf{x})+(k-\underline{k}) \xi_{3}(\mathbf{x})+\xi_{4}(\mathbf{x})=f(\mathbf{x}, k)
$$

for all $k \in[\underline{k}, \bar{k}]$ where

$$
\begin{array}{ll}
\xi_{1}(\mathbf{x})=\alpha^{2}(\mathbf{x}), & \xi_{2}(\mathbf{x})=-\frac{\alpha^{2}(\mathbf{x})\left(A^{2}(\mathbf{x})+1\right)}{2}, \\
\xi_{3}(\mathbf{x})=-2 \alpha(\mathbf{x}) A(\mathbf{x}) \sin (\underline{k} \alpha(\mathbf{x})), & \xi_{4}(\mathbf{x})=A^{2}(\mathbf{x})-2 A(\mathbf{x}) \cos (\underline{k} \alpha(\mathbf{x}))+1 .
\end{array}
$$

Consider now the case $\alpha(\mathbf{x})=0$. Then by 4.8$) \xi_{1}(\mathbf{x})=\xi_{2}(\mathbf{x})=\xi_{3}(\mathbf{x})=0$ and $\xi_{4}(\mathbf{x})=A^{2}(\mathbf{x})-2 A(\mathbf{x})+1$. Hence, it follows from (4.3) that 4.7) remains valid for the case $\alpha(\mathbf{x})=0$.

For each $k \in[\underline{k}, \bar{k}]$, equation (4.7) is a linear equation with respect to the unknown vector $\boldsymbol{\xi}(\mathbf{x})=\left(\xi_{1}(\mathbf{x}), \xi_{2}(\mathbf{x}), \xi_{3}(\mathbf{x}), \xi_{4}(\mathbf{x})\right)$. Consider the partition of the interval $[\underline{k}, \bar{k}]$ with the uniform step size $h=k_{j-1}-k_{j}$ for all $j \in\{1, \ldots, N\}$,

$$
k_{N}=\underline{k}<k_{N-1}<\cdots<k_{1}<k_{0}=\bar{k} .
$$

Then setting $k=k_{j}$ in (4.7), we obtain a linear algebraic system with respect to the vector $\boldsymbol{\xi}$. This system is over-determined since we have only 4 unknowns while $N+1$, the number of equations in the system, is much greater than 4 . We write this over-determined system as

$$
\mathcal{F} \boldsymbol{\xi}=\mathfrak{f}
$$


where the $j^{\text {th }}$ row of the $(N+1) \times 4$ matrix $\mathcal{F}$ is given by $\left(F_{2}\left(\mathbf{x}, k_{j}\right),\left(k_{j}-\underline{k}\right)^{2},\left(k_{j}-\underline{k}\right), 1\right)$ and the $j^{\text {th }}$ component of the $N+1$ dimensional vector $\mathfrak{f}$ is $f\left(\mathbf{x}, k_{j}\right), j=\overline{0, N}$. Then, we solve the following linear algebraic system

$$
\left(\mathcal{F}^{T} \mathcal{F}+\epsilon \mathrm{I}_{4}\right) \xi=\mathcal{F}^{T} \mathfrak{f}
$$

where $\mathcal{F}^{T}$ is $\mathcal{F}$ transpose and $\mathrm{I}_{4}$ is the $4 \times 4$ identity matrix. The positive small number $\epsilon$ plays the role of regularization and its presence guarantees that (4.11) is uniquely solvable. In our computations, the number $\epsilon$ is chosen by a trial and error procedure (Section 6.3). After solving (4.11), we use 4.8) to set:

$$
\alpha(\mathbf{x})=\Re\left(\sqrt{\xi_{1}(\mathbf{x})}\right), \quad \tau(\mathbf{x})=\alpha(\mathbf{x})+x_{3} .
$$

Out of two possible values of $\sqrt{\xi_{1}(\mathbf{x})}$ we take the one for which $\Re\left(\sqrt{\xi_{1}(\mathbf{x})}\right) \geq 0$. After obtaining $\alpha(\mathbf{x})$, we compute $A(\mathbf{x})$ from 4.3 as

$$
A(\mathbf{x})=\left|\cos (k \alpha(\mathbf{x}))+\sqrt{\cos ^{2}(k \alpha(\mathbf{x}))+f(\mathbf{x}, k)-1}\right| .
$$

Let $\tau(\mathbf{x})$ and $A(\mathbf{x})$ be two functions reconstructed by the method of this section. Then following Theorem 3.1, we obtain the following two approximate formulas for $\mathbf{x}=\left(x_{1}, x_{2}, x_{3}\right) \in$ $P_{\text {meas }}$ :

$$
u(\mathbf{x}, k)=A(\mathbf{x}) \exp (\mathrm{i} k \tau(\mathbf{x})), \quad u_{\mathrm{sc}}(\mathbf{x}, k)=A(\mathbf{x}) \exp (\mathrm{i} k \tau(\mathbf{x}))-\exp \left(\mathrm{i} k x_{3}\right)
$$

To illustrate 4.13), we arrange a uniform $100 \times 100$ grid points $\left\{\mathbf{x}_{n}\right\}_{n=1}^{10,000}$ in $P_{\text {meas }}$ and show, in Figure 3 , the true and reconstructed real and imaginary parts of $u_{\mathrm{sc}}\left(\mathbf{x}_{n}, k=\right.$ 82.25) where $n \in\{4800, \ldots, 5100\}$.

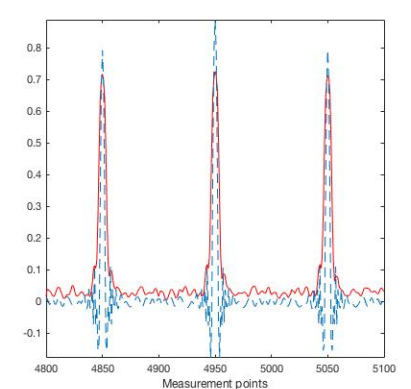

(a) The real parts of the true (solid line) and reconstructed (dashed line) scattered fields.

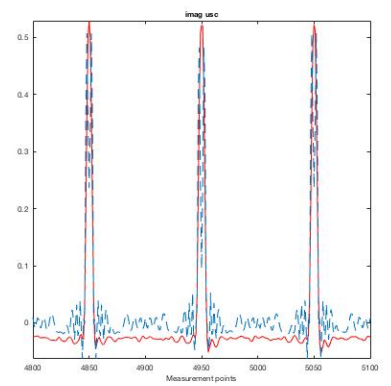

(b) The imaginary parts of the true (solid line) and reconstructed (dashed line) scattered fields.

Figure 3: An example of the reconstructed functions $u_{s c}(\mathbf{x}, k)$. The function $u_{s c}(\mathbf{x}, k)$ is computed by formula (4.13) for $k=82.25$. The data, with $5 \%$ noise, for these computations correspond to Case 1 of Section 6.4.

Remark 4.2. The reconstruction procedure described above is stable due to the stability of the integration with respect to the noise. Indeed, our above analysis is based on the integrals of the data. 
Remark 4.3 (shifting the interval of wave numbers). Formula (4.13) approximates $u_{\mathrm{sc}}(\mathrm{x}, k)$ for $k \in[\underline{k}, \bar{k}]$. However, numerical solution of Helmholtz equation for large values of the wave number $k$ is very computationally expensive. Thus, in our numerical tests, we extend (4.13) to another interval of wave numbers $k \in\left[\underline{k}^{\prime}, \bar{k}^{\prime}\right] \subset(0, \infty)$ with smaller values of $k$, i.e. $\bar{k}^{\prime}<\bar{k}$. To do this, we simply use in (4.13) values $k \in\left[\underline{k}^{\prime}, \bar{k}^{\prime}\right]$. Thus, we first calculate $A(\mathbf{x})$ and $\tau(\mathbf{x})$ using values of $k \in[\underline{k}, \bar{k}]$ from the original interval and then use in (4.13) $k \in\left[\underline{k}^{\prime}, \bar{k}^{\prime}\right]$. We assume everywhere below that this shift is made and, to simplify notations, denote again $\underline{k}^{\prime}:=\underline{k}, \bar{k}^{\prime}:=\bar{k}$.

In Sections 5 and 6, we briefly outline our globally convergent algorithm of [32], which is playing an important role in our method to solve the PCISP.

\section{The phased inverse scattering problem}

We explain in Section 6.2 how to approximately obtain the boundary function $g(\mathbf{x}, k)$ for $\mathbf{x} \in \partial \Omega, k \in[\underline{k}, \bar{k}]$ in 6.3 using (4.13). Hence, our inverse scattering problem becomes now the phased inverse scattering problem:

Problem 5.1 (phased inverse scattering problem). Given

$$
g(\mathbf{x}, k)=u(\mathbf{x}, k), \quad \mathbf{x} \in \partial \Omega, k \in[\underline{k}, \bar{k}],
$$

where $u(\mathbf{x}, k)$ is the solution of (2.6), determine the function $c(\mathbf{x})$ for $\mathbf{x} \in \Omega$.

The inverse problem (5.1) has a broad range of applications and has been widely studied. As to its uniqueness, we refer to item 2 in Remarks 2.2. We refer to [?,2, 4, 8, 14, 32, 36, 37, 42, 43 and references therein for various studies of numerical methods and reconstruction procedures for solving this inverse problem under a variety of assumptions on the measurement setup. The globally convergent algorithm of [32] has been developed to solve the inverse problem (5.1) with only a single measurement of multi-frequency scattering data. In addition, we refer to [28, 34 and references cited therein for the second globally convergent numerical method for the single measurement case, which is based on the construction of weighted globally strictly convex Tikhonov-like functionals with Carleman weight functions in them.

Below in this section we briefly describe the globally convergent numerical method of [32]. We refer to [32] for details, which, in particular, include the global convergence theorem 6.1.

\subsection{An integro-differential equation}

In this section, we assume that the function $u(\mathbf{x}, k)$ never vanishes. This assumption is true when $k$ is large due to (4.1), see [32] for more details. Since the vector $\nabla u(\mathbf{x}, k) / u(\mathbf{x}, k)$ is curl free, we can follow a procedure in [32, Lemma 4.1] to find a smooth function $v(\mathbf{x}, k)$ such that

$$
\exp (v(\mathbf{x}, k))=u(\mathbf{x}, k), \quad \nabla v(\mathbf{x}, k)=\frac{\nabla u(\mathbf{x}, k)}{u(\mathbf{x}, k)} \quad \mathbf{x} \in \Omega, k \in[\underline{k}, \bar{k}] .
$$


The function $v$ can be understood as the natural logarithm of the function $u$. It satisfies

$$
\Delta v(\mathbf{x}, k)+(\nabla v(\mathbf{x}, k))^{2}=-k^{2} c(\mathbf{x}), \quad \mathbf{x} \in \Omega, k \in[\underline{k}, \bar{k}] .
$$

Defining

$$
q(\mathbf{x}, k)=\frac{\partial v(\mathbf{x}, k)}{\partial k}, \quad \mathbf{x} \in \Omega, k \in[\underline{k}, \bar{k}]
$$

and differentiating (5.3) with respect to $k$, we obtain that the function $q(\mathbf{x}, k)$ satisfies

$$
\begin{aligned}
& \frac{k}{2} \Delta q(\mathbf{x}, k)+k \nabla q(\mathbf{x}, k) \cdot\left(-\int_{k}^{\bar{k}} \nabla q(\mathbf{x}, s) s+\nabla V(\mathbf{x})\right) \\
& \quad=-\int_{k}^{\bar{k}} \Delta q(\mathbf{x}, s) s+\Delta V(\mathbf{x})+\left(-\int_{k}^{\bar{k}} \nabla q(\mathbf{x}, s) s+\nabla V(\mathbf{x})\right)^{2}, \mathbf{x} \in \Omega, k \in[\underline{k}, \bar{k}]
\end{aligned}
$$

and that, due to $(5.2), q(\mathbf{x}, k)$ satisfies the following Dirichlet boundary condition

$$
q(\mathbf{x}, k)=\frac{\partial_{k} u(\mathbf{x}, k)}{u(\mathbf{x}, k)} \quad \mathbf{x} \in \partial \Omega .
$$

The function $V(\mathbf{x})$ is named the tail function.

\subsubsection{The initial approximation $V_{0}(\mathrm{x})$ for the tail function}

Solving Problem 5.1 is somewhat equivalent to finding the function $q(\mathbf{x}, k), \mathbf{x} \in \Omega$. Therefore, solving (5.4)-(5.5) is crucial. However, the vector function $\nabla V(\mathbf{x})$ is involved in equation (5.4), where the tail function $V(\mathbf{x})$ is still unknown. In this subsection, following [32], we provide an initial approximation for $\nabla V(\mathbf{x})$ and denote this approximation $\nabla V_{0}(\mathbf{x})$. Thus, $\nabla V_{0}(\mathbf{x})$ is an important ingredient of the global convergence theorem of [32]. We note that computing $\nabla V_{0}(\mathbf{x})$ does not require any a priori knowledge of a good initial guess for the true solution of Problem 5.1. This is unlike conventional locally convergent numerical methods.

Recall that the tail function is defined as $V(\mathbf{x})=v(\mathbf{x}, \bar{k})$. Assuming that numbers $\underline{k}$ and $\bar{k}$ are sufficiently large, dropping the term $O(1 / k)$ in $(4.1)$ and using $(5.2)$, we obtain

$$
V(\mathbf{x}) \approx \ln A(\mathbf{x})+i \bar{k} \tau(\mathbf{x})=i \bar{k} \tau(\mathbf{x})\left(1+\frac{\ln A(\mathbf{x})}{i \bar{k} \tau(\mathbf{x})}\right) \approx i \bar{k} \tau(\mathbf{x}), \quad \mathbf{x} \in \Omega
$$

Therefore, the function $q(\mathbf{x}, \bar{k})$ can be approximated as

$$
q(\mathbf{x}, \bar{k})=\left.\partial_{k} v(\mathbf{x}, k)\right|_{k=\bar{k}} \approx i \tau(\mathbf{x}) \approx \frac{V(\mathbf{x})}{\bar{k}}, \quad \mathbf{x} \in \Omega
$$

Substituting (5.6) into (5.4) and setting in (5.4) $k=\bar{k}$, we obtain

$$
\frac{1}{2} \Delta V(\mathbf{x})+(\nabla V(\mathbf{x}))^{2}=\Delta V(\mathbf{x})+(\nabla V(\mathbf{x}))^{2}, \quad \mathbf{x} \in \Omega
$$


which yields

$$
\Delta V(\mathbf{x})=0, \quad \mathbf{x} \in \Omega .
$$

Note that only $\nabla V(\mathbf{x})$ and $\Delta V(\mathbf{x})=\operatorname{div}(\nabla V(\mathbf{x}))$ are involved in equation (5.4). Hence, rather that instead finding $V(\mathbf{x})$, we compute directly the vector function $\nabla V(\mathbf{x})$ in our numerical implementation. To do this, we solve the following problem

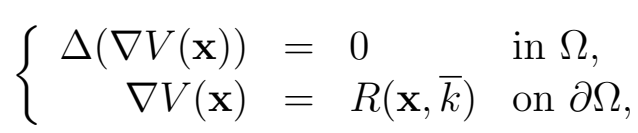

where $R(\mathbf{x}, \bar{k})$ is a certain vector function, which is known approximately. We refer to 32 , Section 7.4] for all the details about the approximation of $R(\mathbf{x}, \bar{k})$ on the entire boundary $\partial \Omega$ using, in particular, (5.1) and (6.3). We consider the solution of problem (5.7) as the first approximation $\nabla V_{0}$ of the vector function $\nabla V$. We mention once again that the globally convergent numerical method outlined in this Section 5, including the approximation of $[32$, Section 7.4] for the vector function $R(\mathbf{x}, \bar{k})$, has worked quite well for the microwave experimental data, see references in Section 1.

\subsubsection{The globally convergent algorithm}

For $N \in \mathbb{N}$, consider the uniform partition

$$
k_{N}=\underline{k}<k_{N-1}<\cdots<k_{1}<k_{0}=\bar{k}
$$

of the interval $[\underline{k}, \bar{k}]$ with the step size $h=k_{i-1}-k_{i}, 1 \leq i \leq N$. Although this partition is different from the one in (4.9), we keep the same notation here for brevity. For each $n \in\{1, \ldots, N\}$, denote

$$
q_{n}(\mathbf{x})=q\left(\mathbf{x}, k_{n}\right), \quad u_{n}(\mathbf{x})=u\left(\mathbf{x}, k_{n}\right), \quad \mathbf{x} \in \Omega .
$$

Recall that the first approximation $\nabla V_{0}$ for the gradient $\nabla V$ of the tail function is constructed in Section 5.1.1. We assume, inductively, that $\nabla V_{n-1}$ is known, which implies that $\Delta V_{n-1}=\operatorname{div}\left(\nabla V_{n-1}\right)$ is known as well, where $n \in\{1, \ldots, N\}$. By (5.8) and (5.9) the discrete, with respect to $k$, form of equation (5.4) is

$$
\begin{aligned}
k_{n} \Delta q_{n}(\mathbf{x}) & -2 k_{n} \nabla q_{n}(\mathbf{x}) \cdot \nabla Q_{n-1}(\mathbf{x})+2 k_{n} \nabla q_{n} \cdot \nabla V_{n-1}(\mathbf{x}) \\
= & -2 \Delta Q_{n-1}(\mathbf{x})+2 \Delta V_{n-1}(\mathbf{x})+2\left(-\nabla Q_{n-1}(\mathbf{x})+\nabla V_{n-1}(\mathbf{x})\right)^{2}, \quad \mathbf{x} \in \Omega
\end{aligned}
$$

where

$$
Q_{n-1}(\mathbf{x})=h \sum_{i=0}^{n-1} q_{n}(\mathbf{x}), \quad \mathbf{x} \in \Omega .
$$

Here, we approximate the integral $\int_{k}^{\bar{k}} q(\mathbf{x}, s) d s$ by $Q_{n-1}(\mathbf{x})$ instead of $Q_{n}(\mathbf{x})$ to remove the nonlinearity of (5.4). The resulting error is $O(h)$, as $h \rightarrow 0$. The boundary condition for the function $q_{n}(\mathbf{x})$ is

$$
q_{n}(\mathbf{x})=\frac{g\left(\mathbf{x}, k_{n}\right)-g\left(\mathbf{x}, k_{n+1}\right)}{h g\left(\mathbf{x}, k_{n}\right)}, \quad \mathbf{x} \in \partial \Omega .
$$


Remark 5.1. Thus, (5.10)-(5.12) is the Dirichlet boundary value problem for an elliptic equation (5.10). In [32], for the theoretical purpose, we make one more approximation for equation (5.10) via replacing the term $2 k_{n} \nabla q_{n} \cdot \nabla V_{n-1}(\mathbf{x})$ with the term $2 k_{n} \nabla q_{n-1}$. $\nabla V_{n-1}(\mathbf{x})$. The resulting error is still $O(h)$ as $h \rightarrow 0$. In this paper, although we skip this approximation and use (5.10) to calculate the function $q_{n}$, the numerical results are still accurate, see Section 6 .

Remark 5.2. Although the derivative of the data with respect to $k$ is calculated in (5.12) via the finite difference, we have not observed any instabilities in our computations, probably because the step size $h$ was not exceedingly small. The same is true for all above cited publications about the globally convergent numerical methods of this group.

In short, the algorithm is based on the following iterative process: (1) given $\nabla V_{n-1}$, $\Delta V_{n-1}$, solve the Dirichlet boundary value problem (5.10)-(5.12); (2) use $(5.3)$ at $k:=\underline{k}$ to calculate the function $c_{n}(\mathbf{x})$ via $q_{n}(\mathbf{x}), \nabla V_{n-1}(\mathbf{x})$ and $\Delta V_{n-1}(\mathbf{x}) ;(3)$ update $\nabla V_{n}(\mathbf{x})$ and $\Delta V_{n}(\mathbf{x})$ by solving the Lippmann-Schwinger equation 2.11$)$ with $\beta(\mathbf{x}):=c_{n}(\mathbf{x})-1$. To increase the stability of this iterative process, we arrange internal iterations inside steps (1)-(3). The whole algorithm is summarized as follows, see [32 for more details:

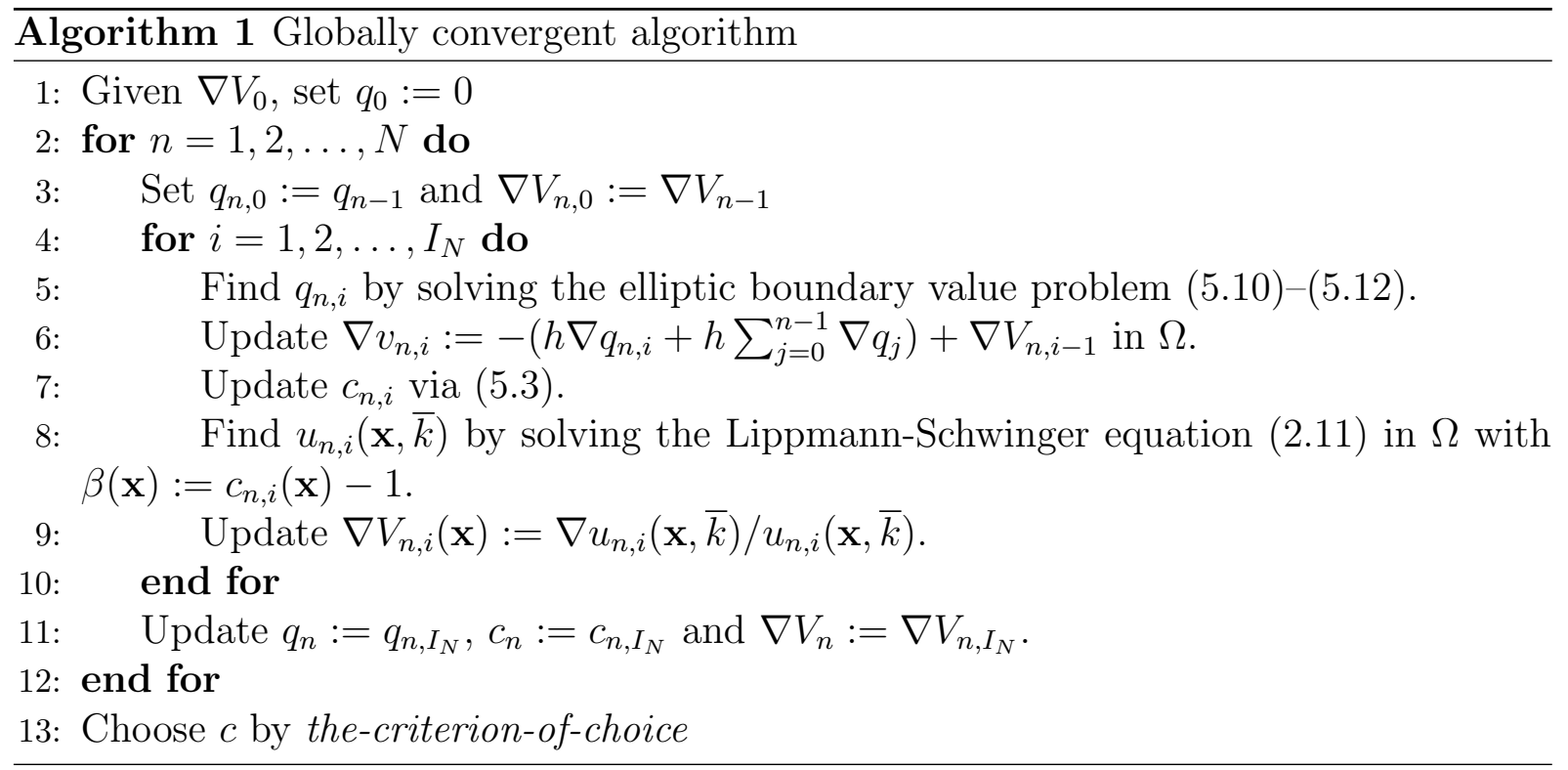

Remark 5.3. The stopping rule for the iterative loops in Algorithm 1 is presented in [32, $33,40,41]$.

\section{$6 \quad$ Numerical studies}

\subsection{Summary of our method for solving the PCISP}

In this section, we summarize the whole procedure of the reconstruction of the coefficient $c(\mathbf{x})$ from the knowledge of $\left|u_{\mathrm{sc}}(\mathbf{x}, k)\right|, \mathbf{x} \in P_{\text {meas }}$ and $k \in[\underline{k}, \bar{k}]$ as follows: 
Algorithm 2 Globally convergent algorithm for the phaseless inverse scattering problem

1: For each point $\mathbf{x}$ in $P_{\text {meas }}$ find numbers $\tau(\mathbf{x})$ and $A(\mathbf{x})$ as described in section 4. Next, approximate the function $u_{\mathrm{sc}}(\mathbf{x}, k)$ via $\sqrt{4.13}$ for $\mathrm{x} \in P_{\text {meas }}, k \in[\underline{k}, \bar{k}]$, where $[\underline{k}, \bar{k}]$ is the "shifted" interval of wave numbers as in Remark 4.3 .

2: Propagate the reconstructed $u_{\mathrm{sc}}(\mathbf{x}, k)$ from $P_{\text {meas }}$ to the plane $P_{\text {prop }}$, see Section 6.2. The plane $P_{\text {prop }}$ is closer to the targets than $P_{\text {meas }}$.

3: Consider the square $\Gamma \subset \partial \Omega$ in $(6.2)$, which is a part of the propagated plane $P_{\text {prop }}$, where $\Omega$ is the domain of our interest containing all targets. Assign the data on $\partial \Omega$ as in $(6.3)$.

4: Having approximated the function $u(\mathbf{x}, k)$ on $\partial \Omega$ as in $(6.3)$, find $c(\mathbf{x})$ by Algorithm 1 .

\subsection{Data propagation and completion}

The data propagation is a procedure which enables us to "move" the data to a plane which is closer to the target than the original measurement plane. Thus, we "propagate" the reconstructed function $u_{\mathrm{sc}}$ in $(4.13)$ from the square $P_{\text {meas }} \subset P$ to $P_{\text {prop }}$. Here $P_{\text {meas }}$ is the square (2.7) on the measurement plane $P=\left\{\mathbf{x}: x_{3}=R\right\}$ and $P_{\text {prop }}$ is a propagated plane which is closer to the targets of our interest. This data propagation process has been rigorously justified in [40]. By our experience in the previous works [33,40,41, this process enables one not only to propagate the scattered wave but also to significantly decrease the amount of noise in the data. Moreover, unlike the measured data, the propagated data focuses more at the $x_{1}, x_{2}$ positions of the targets. We briefly outline the data propagation method here.

Let the number $R^{\prime} \in(0, R)$. Assume that the domain $\Omega \subset\left\{x_{3} \in\left(0, R^{\prime}\right)\right\}$. So, we want to propagate the function $u_{s c}(\mathbf{x}, k)$ given in 4.13 for $k \in[\underline{k}, \bar{k}]$ from $P_{\text {meas }}$ to the plane $P_{\text {prop }}=\left\{x_{3}=R^{\prime}\right\}$. For any pair of real numbers $k_{x_{1}}, k_{x_{2}}$, define

$$
\widehat{u}_{\mathrm{sc}}\left(k_{x_{1}}, k_{x_{2}}, k\right)=\frac{1}{2 \pi} \int_{\mathbb{R}^{2}} u_{\mathrm{sc}}\left(x_{1}, x_{2}, R\right) \exp \left(\mathrm{i}\left(k_{x_{1}} x_{1}+k_{x_{2}} x_{2}\right)\right) d x_{1} d x_{2} .
$$

Here, we have extended $u_{\mathrm{sc}}(\mathbf{x}, k)$ by zero for $\mathbf{x}=\left(x_{1}, x_{2}, R\right) \notin P_{\text {meas }}$. Then it was proved in [40] that the propagated wave field $u_{\mathrm{sc}}(\mathbf{x}, k)$ for $0<R^{\prime}<R$ is given by

$$
u_{\mathrm{sc}}(\mathbf{x}, k)=\frac{1}{2 \pi} \int_{\left\{k_{x_{1}}^{2}+k_{x_{2}}^{2}<k^{2}\right\}} \widehat{u}_{\mathrm{sc}}\left(k_{x_{1}}, k_{x_{2}}, k\right) \exp \left(-\mathrm{i}\left(k_{x_{1}} x_{1}+k_{x_{2}} x_{2}-k_{x_{3}}\left(R^{\prime}-R\right)\right)\right) d k_{x_{1}} d k_{x_{2}}
$$

where $\mathbf{x}=\left(x_{1}, x_{2}, R^{\prime}\right), k_{x_{3}}=\left(k^{2}-k_{x_{1}}^{2}-k_{x_{2}}^{2}\right)^{\frac{1}{2}}$ and $k \in[\underline{k}, \underline{k}]$. We use the same square on the plane $P_{\text {prop }}$ as in $P_{\text {meas }}$ (see (2.7) ) and we do not count values of the function $u_{\mathrm{sc}}(\mathbf{x}, k)$ in (6.1) outside of this square. Thus, we denote that square on $P_{\text {prop }}$ as

$$
\Gamma=\left\{\mathbf{x}:\left|x_{1}\right|<b,\left|x_{2}\right|<b, x_{3}=R^{\prime}\right\} .
$$

We assume that $\Gamma \subset \partial \Omega$.

The data for our globally convergent algorithm are $\left.u(\mathbf{x}, k)\right|_{\partial \Omega \times\left[k^{\prime}, \bar{k}^{\prime}\right]}[32]$. Therefore, we need to complement the data on $\partial \Omega \backslash \Gamma$ as it was done in $[32]$. We are doing so 
heuristically by simply setting $u_{\mathrm{sc}}(\mathbf{x}, k)=0$ for $\mathbf{x} \in \partial \Omega \backslash \Gamma$. In other words, the input $\left.u(\mathbf{x}, k)\right|_{\partial \Omega}:=g(\mathbf{x}, k)$ for the globally convergent numerical method of [32] described above is given by

$$
g(\mathbf{x}, k)= \begin{cases}u_{\mathrm{sc}}(\mathbf{x}, k)+\exp \left(\mathrm{i} k x_{3}\right), & \mathbf{x} \in \Gamma, k \in[\underline{k}, \bar{k}], \\ \exp \left(\mathrm{i} k x_{3}\right), & \mathbf{x} \in \partial \Omega \backslash \Gamma, k \in[\underline{k}, \bar{k}],\end{cases}
$$

see Remark 4.3 for $[\underline{k}, \bar{k}]$.

Remark 6.1. It was shown in subsections 7.6 and 7.7 of [32] that in the case when the correct computationally simulated data are assigned on the entire boundary $\partial \Omega$, the computational result is about the same as for the case when the boundary data given on a part of the boundary are complemented as in (6.3). Also, it was demonstrated in all our above cited works on experimental data that (6.3) works well.

\subsection{Some details of numerical experiments}

Our numerical studies are conducted for a realistic range of parameters which we have extensively discussed with Professor Vasily Astratov from Center for Optoelectronics and Optical Communications of the University of North Carolina at Charlotte.

In at least one experimental arrangement one wants to image dielectric balls whose diameters are about $5 \mu \mathrm{m}$. These balls are called "microspheres". In our computations, the measurement plane is about $25 \mu \mathrm{m}$ away from the domain $\Omega$ where these microspheres are located. The size of the measurement square $P_{\text {meas }}$ is $100 \mu \mathrm{m} \times 100 \mu \mathrm{m}$. The wavelengths of light $\lambda \in[738,785] \mathrm{nm}=[0.738,0.785] \mu \mathrm{m}$.

To make variables dimensionless, we consider the change of variables $\mathbf{x}^{\prime}=\mathbf{x} / 10 \mu \mathrm{m}$ while leaving the same notations for brevity. Then the dimensionless wave number $k=20 \pi / \lambda^{\prime}$, where $\lambda^{\prime}$ is the dimensionless wavelength. Hence, we obtain the interval for the dimensionless $k \in[80,85]=[20 \pi / 0.785,20 \pi / 0.738]$. We then "shift" the interval of wavelength to $[20.4,21]$ as in Remark 4.3 to make the whole procedure less computationally expensive. More precisely, we consider the following setup:

(a) The scattering balls are located near the $x_{1} x_{2}$-plane and their diameter is 0.5 .

(b) The domain $\Omega=(-2.5,2.5) \times(-2.5,2.5) \times(-4,1)$.

(c) The measurement square is $P_{\text {meas }}=\left\{\mathbf{x}:\left|x_{1}\right|,\left|x_{2}\right| \leq 5, x_{3}=2.5\right\}$.

(d) The propagated plane in Step 2 in Algorithm 2 is

$$
P_{\text {prop }}=\left\{\mathbf{x}=\left(x_{1}, x_{2}, x_{3}\right):\left|x_{1}\right|,\left|x_{2}\right| \leq 5, x_{3}=1\right\} .
$$

Then, we choose $\Gamma$ to be a subset of $P_{\text {prop }}$ as

$$
\Gamma=\left\{\mathbf{x}=\left(x_{1}, x_{2}, x_{3}\right):\left|x_{1}\right|,\left|x_{2}\right| \leq 2.5, x_{3}=1\right\} .
$$

The main reason for working with $\Gamma$ instead of $P_{\text {prop }}$ is that the data on $P_{\text {prop }} \backslash \Gamma$ are small and do not contribute the inversion process. Furthermore, this choice leads to a smaller computational domain $\Omega$. Thus, in notations of Section $6.2, R=2$ and $R^{\prime}=1$. We had $100 \times 100$ uniform grid $\left\{\mathbf{x}_{n}\right\}$ covering the square $P_{\text {meas }}$. Functions $A$ and $\tau$ were 
reconstructed at these grid points. We have chosen the regularization parameter $\epsilon=0.03$ in (4.11) by trial and error. Thus, we have obtained numbers $A\left(\mathbf{x}_{n}\right)$ and $\tau\left(\mathbf{x}_{n}\right)$. These numbers were reconstructed from noisy data. For $k \in[80,85]$ the $5 \%$ random noise was introduced as:

$$
f_{\text {noise }}(\mathbf{x}, k)=f(\mathbf{x}, k)+5 \%\|f\|_{L^{2}\left(P_{\text {meas }} \times[\underline{k}, \bar{k}]\right)} \operatorname{rand}(\mathbf{x}, k) /\|\operatorname{rand}\|_{L^{2}\left(P_{\text {meas }} \times[\underline{k}, \bar{k}]\right)}
$$

for $\mathbf{x} \in P_{\text {meas }}, k \in[\underline{k}, \bar{k}]$ where $\operatorname{rand}(\mathbf{x}, k)$ is a random number in $(0,1)$.

\subsection{Numerical results}

To make our spherical inclusions to be smoothly embedded in the background medium, we consider the following construction. Let $B\left(\mathbf{x}_{0}, r\right)$ be the ball of the radius $r$ centered at the point $\mathbf{x}_{0} \in \mathbb{R}^{3}$. Define the function $\chi_{B\left(\mathbf{x}_{0}, r\right)}(\mathbf{x})$,

$$
\chi_{B\left(\mathbf{x}_{0}, r\right)}(\mathbf{x})= \begin{cases}\exp \left(1-r^{2} /\left(r^{2}-\left|\mathbf{x}-\mathbf{x}_{0}\right|^{2}\right)\right) & \mathbf{x} \in B\left(\mathbf{x}_{0}, r\right) \\ 0 & \mathbf{x} \in \mathbb{R}^{3} \backslash \overline{B\left(\mathbf{x}_{0}, r\right)} .\end{cases}
$$

Hence, the function $\chi_{B\left(\mathbf{x}_{0}, r\right)} \in C^{\infty}\left(\mathbb{R}^{3}\right)$ and its support is $B\left(\mathbf{x}_{0}, r\right)$. We present below the following three cases of the numerical reconstruction of the functions $c(\mathbf{x})$ :

1. One inclusion: $c(\mathbf{x})=1+\chi_{B\left(\mathbf{x}_{0}, r\right)}$ where $r=0.25$ and $\mathbf{x}_{0}=(0,0,0.25)$.

2. Two inclusions, which are symmetric with respect to the plane $\left\{x_{1}=0\right\}: c(\mathbf{x})=$ $1+\chi_{B\left(\mathbf{x}^{(1)}, r\right)}+\chi_{B\left(\mathbf{x}^{(2)}, r\right)}$ where $r=0.25, \mathbf{x}^{(1)}=(-0.5,0,0.25)$ and $\mathbf{x}^{(2)}=(0.5,0,0.25)$.

3. Two inclusions which are non-symmetric with respect to the plane $\left\{x_{2}=0\right\}$ but symmetric with respect to the plane $\left\{x_{1}=0\right\}: c(\mathbf{x})=1+\chi_{B\left(\mathbf{x}^{(3)}, r\right)}+\chi_{B\left(\mathbf{x}^{(4)}, r\right)}$ where $r=0.25, \mathbf{x}^{(3)}=(0.5,0.5,0.25)$ and $\mathbf{x}^{(4)}=(-0.5,-0.25,0.25)$.

Here, an inclusion means a connected component of the support of the function $c(\mathbf{x})-1$. In labels for Figures $4,6 c^{*}(\mathbf{x})$ and $c_{\text {comp }}(\mathbf{x})$ mean the exact and computed coefficients $c(\mathbf{x})$, respectively. We display in these figures:

(a) A 2D cross-sectional view of the true inclusions through their center by a plane which is orthogonal to the $x_{1}, x_{2}$-plane.

(b) A $3 \mathrm{D}$ view of the true inclusions by isosurfaces.

(c) A $2 \mathrm{D}$ cross-sectional view of the reconstructed inclusions on the same plane as in (a).

(d) A 3D view of the reconstructed inclusions by isosurfaces. 


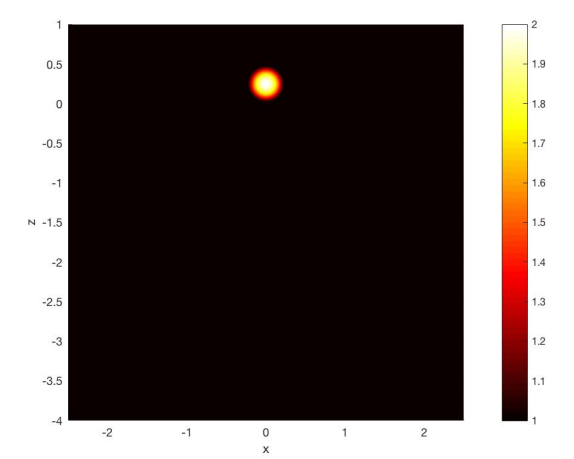

(a) Exact profile (cross-sectional view)

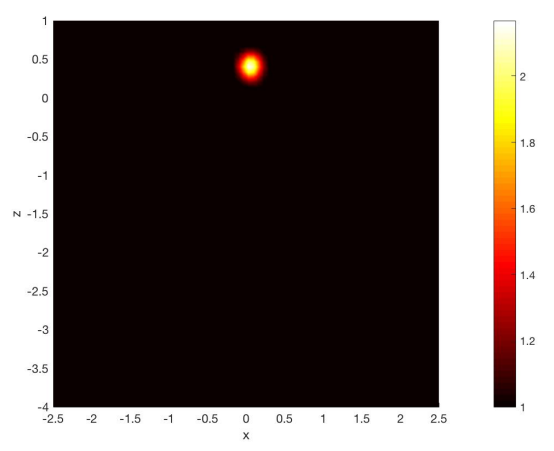

(c) Reconstruction (cross-sectional view)

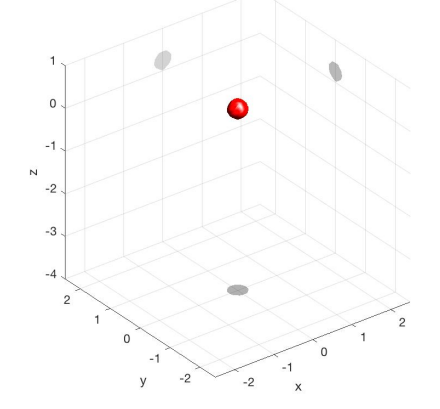

(b) Exact profile (3D view)

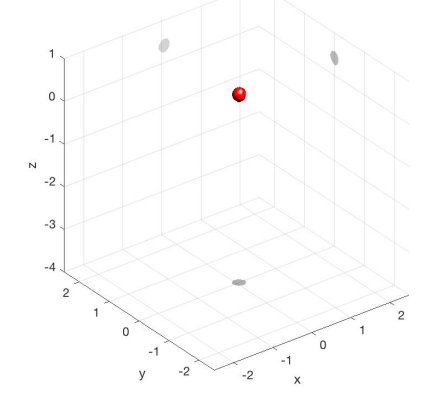

(d) Reconstruction (3D view)

Figure 4: Reconstruction results for the case of one spherical inclusion: the above item 1. The maximal value of $c^{*}(\mathbf{x})$ in this inclusion is 2. The maximal value of $c_{c o m p}(\mathbf{x})$ in this inclusion is 2.16. Hence, the error in computing this value is $8 \%$

\section{Summary}

In this paper, we have developed a numerical method for solving a 3D phaseless inverse scattering problem. Unlike the previous work [20] of this group, where overdetermined data were used and a linearization of the travel time information was applied, we work here with the data generated by a single measurement event using a single direction of the incident plane wave and an interval of frequencies. Our procedure consists of two stages and it does not use any linearization. On the first stage we reconstruct the first term of the asymptotic expansion at $k \rightarrow \infty$ of the function $u_{\mathrm{sc}}(\mathbf{x}, k)$ for $\mathbf{x} \in P_{\text {meas }}$. As a result, we obtain a phased coefficient inverse scattering problem.

On the second stage, we solve the latter problem by the globally convergent numerical method of [32]. Our results demonstrate a good reconstruction accuracy of locations of abnormalities. In addition, the relative errors in abnormality/background contrasts, which are the maximal values of the computed coefficients $c_{\text {comp }}(\mathbf{x})$ of abnormalities, do not exceed $8 \%$ in all cases. Given a significant complexity of the problem under consideration and 5\% random noise in the data, we consider this as a quite accurate result.

As to the theoretical part, we prove here uniqueness Theorem 3.2 which claims that the first term of the asymptotic expansion at $k \rightarrow \infty$ of the function $u_{\mathrm{sc}}(\mathbf{x}, k)$ can be 


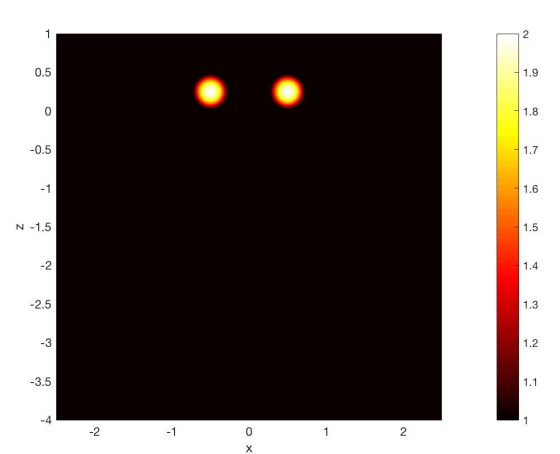

(a) Exact profile (cross-sectional view)

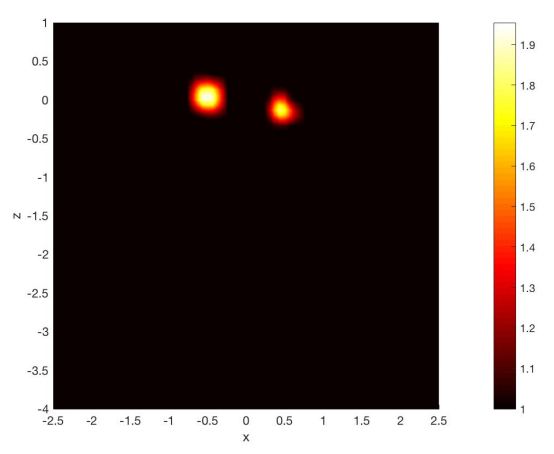

(c) Reconstruction (cross-sectional view)

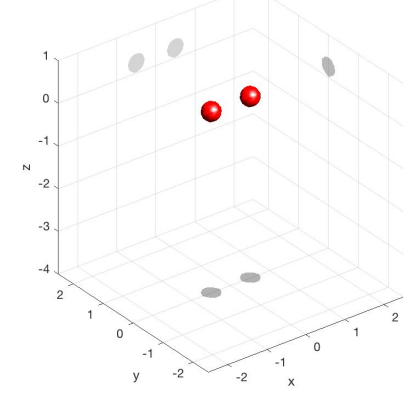

(b) Exact profile (3D view)

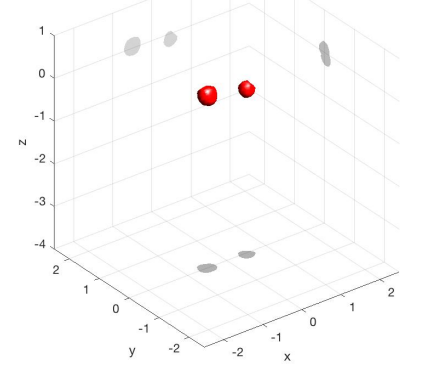

(d) Reconstruction (3D view)

Figure 5: Reconstruction results for two spherical inclusions: the above item 2. The maximal value of $c^{*}(\mathbf{x})$ in both inclusions are 2. Maximal value of the computed $c_{\text {comp }}(\mathbf{x})$ is 1.95 in both inclusions. Hence, the error in computing this value is $2.5 \%$

uniquely reconstructed from our phaseless data.

\section{Appendix - The full Maxwell's equations and the scalar 3D Helmholtz equation}

In this section we present some numerical simulations, which aim to show that, under some assumptions, the solution of the scalar 3D Helmholtz equation can be used to approximate such a component of the electric field satisfying the Maxwell's equations, which is originally incident upon the medium. We consider two cases here: the backscatter data and the forward scatter data.

For the backscatter data, we want to verify the use of the Helmholtz equation in our paper [32] as well as in our works on experimental microwave data [33, 40, 41]. Therefore, we follow the same setup as in [32]. As to the forward scatter data, we test them to verify the model problem studied in this paper.

Assume that the scattering objects are isotropic, non-magnetic and that they are characterized by the dielectric constant $c(\mathbf{x})$, which is a bounded real-valued function satisfying

$$
c(\mathbf{x}) \geq 1 \text { for all } \mathbf{x} \in \mathbb{R}^{3} \quad \text { and } c(\mathbf{x})=1 \text { for all } \mathbf{x} \in \mathbb{R}^{3} \backslash \Omega \text {. }
$$




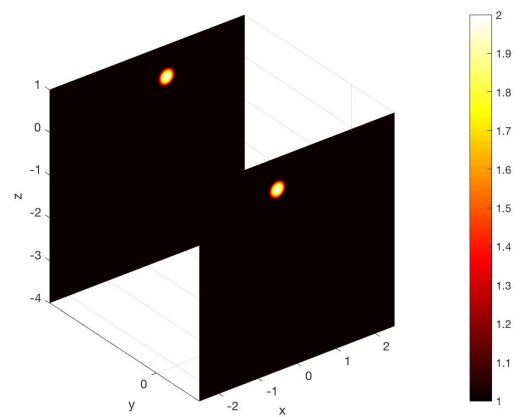

(a) Exact profile (cross-sectional view)

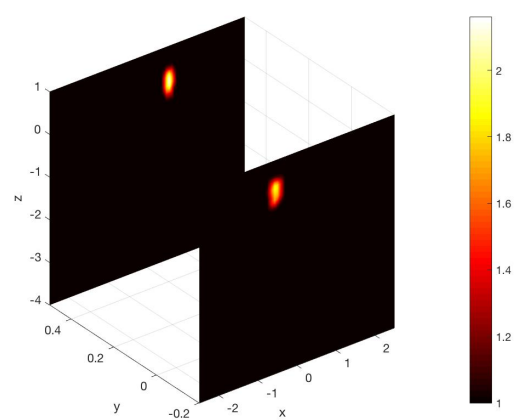

(c) Reconstruction (cross-sectional view)

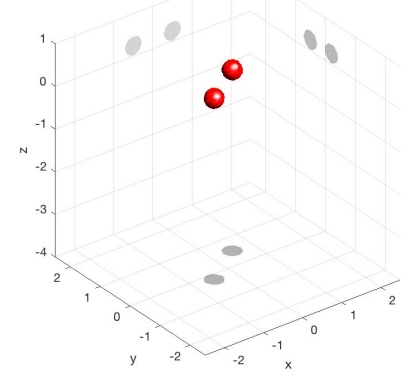

(b) Exact profile (3D view)

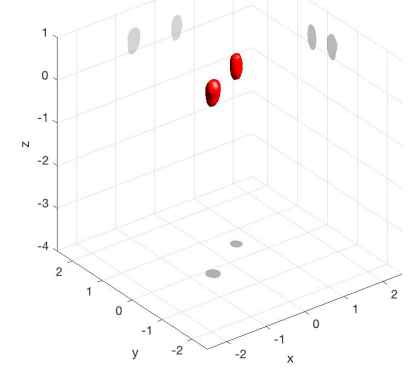

(d) Reconstruction (3D view)

Figure 6: Reconstruction results for two non symmetric spherical inclusions: the above item 3. The maximal value of $c^{*}(\mathbf{x})$ in both inclusions is 2. The maximal value of the computed coefficient $c_{\text {comp }}(\mathbf{x})$ is 1.91 and 2.16 in the left and right inclusions respectively. Hence, the error in computing this value is $4.5 \%$ and $8 \%$ respectively.

Let $\mathbf{E}$ be the electric field. The scattering of light in the frequency domain can be described by the Maxwell's equations for the electric field as follows:

$$
\begin{aligned}
& \nabla \times \nabla \times \mathbf{E}-k^{2} c(\mathbf{x}) \mathbf{E}=0, \quad \mathbf{x} \in \mathbb{R}^{3}, \\
& \mathbf{E}(\mathbf{x}, k)=\mathbf{E}^{\mathrm{inc}}(\mathbf{x}, k)+\mathbf{E}^{\mathrm{sc}}(\mathbf{x}, k), \\
& \lim _{|\mathbf{x}| \rightarrow \infty}|\mathbf{x}|\left(\nabla \times \mathbf{E}^{\mathrm{sc}} \times \hat{\mathbf{x}}-i k \mathbf{E}^{\mathrm{sc}}\right)=0, \quad \hat{\mathbf{x}}=\mathbf{x} /|\mathbf{x}| .
\end{aligned}
$$

The total electric field $\mathbf{E}$ is the sum of the scattered field $\mathbf{E}^{\mathrm{sc}}=\left(E_{1}^{\mathrm{sc}}, E_{2}^{\mathrm{sc}}, E_{3}^{\mathrm{sc}}\right)$ and the incident field $\mathbf{E}^{\text {inc }}$. We note that the scattered field $\mathbf{E}^{\mathrm{sc}}$ satisfies the Silver-Muller radiation condition (8.3), which guarantees that it is an outgoing wave.

We now consider the scattering problem for the scalar Helmholtz equation as in [32], where the incident wave is a plane wave propagating along the $z$-direction

$$
\begin{aligned}
& \Delta u+k^{2} c(\mathbf{x}) u=0, \quad \mathbf{x} \in \mathbb{R}^{3}, \\
& u=e^{i k z}+u^{\mathrm{sc}}, \\
& \lim _{r \rightarrow \infty} r\left(\partial_{r} u^{\mathrm{sc}}-i k u^{\mathrm{sc}}\right)=0, \quad r=|\mathbf{x}| .
\end{aligned}
$$

The measurement square for the backscatter data is $\left\{\mathbf{x}:\left|x_{1}\right|,\left|x_{2}\right| \leq 5, x_{3}=-10\right\}$, 
while the face of the scatterer is at $\{z=0\}$. For the forward scatter data, we measure at the same square $P_{\text {meas }}$ as in Section $6.3 P_{\text {meas }}=\left\{\mathbf{x}:\left|x_{1}\right|,\left|x_{2}\right| \leq 5, x_{3}=2.5\right\}$.

We observed in our numerical simulation that if the incident field in (8.1) $-(8.3)$ $\mathbf{E}^{\text {inc }}(\mathbf{x}, k)=(0,1,0) e^{i k x_{3}}$, then the second component of the electric scattered field $E_{2}^{\text {sc }}$ on the measurement square can be well-approximated by the scattered field of the Helmholtz problem $u_{\text {sc }}$ divided by a scalar multiplier $d(k)$, defined by

$$
d(k)=\frac{\max \left(\left|u_{\mathrm{sc}}(\mathbf{x}, k)\right|\right)}{\max \left(\left|E_{2}^{\mathrm{sc}}(\mathbf{x}, k)\right|\right)},
$$

where the maximal values are taken on the measurement square.

The observation above means that the experimental scattering data studied in [32,41], which are supposed to be the second component of the electric scattered field, can be calibrated and approximated by the solution to the scattering problem for the scalar Helmholtz equation. The solution of Maxwell problem 8.1)-8.3 and the scalar problem (8.4)-(8.6) was computed using the numerical solvers developed in [35] and [39], respectively.

Now we present four numerical examples. In the first two examples (Figures 7 and 8), we consider the backscatter data and the setup in [32]. More precisely, we consider wave numbers $k=6.5$ and $k=7.5$, the measurement square $[-5,5]^{2}$ is uniformly discretized by $50^{2}$ points. To show the fitting of the data in our simulations, we first transform the $50 \times 50$ data matrix in a vector of 2500 points (using the command : (colon) in MATLAB). Then we choose the data points from 1200 to 1300, where the signals of the scattered fields are the strongest, to present them in Figures 7 and 8 . We note that the results are similar for other measurement points, where the scattered fields are weaker. Figure 7 is dedicated to the case of a spherical scattering object represented by a smooth function $c(\mathbf{x})$, which is similar to the model in Section 6.4 except $\max \{c(\mathbf{x})\}=4.5$ instead of 2. We consider in Figure 8 the case of a rectangular scattering object represented by a piecewise constant function $c(\mathbf{x})$ with jumps across the boundary of the scatterer $(c(\mathbf{x})=4.5$ inside the scatterer).

In Figure 9 and Figure 10 we present the numerical simulations for the setup considered in Section 6.4. More precisely, we consider the case of one inclusion and the case of two inclusions there, where wave number $k$ is 20.5 and 21.5. We have $100^{2}$ measurement points and choose to present the points from 4900 to 5100 , where scattered fields seem to be strongest.

\section{Acknowledgements}

This work was supported by the Office of Naval Research grant N00014-15-1-2330 as well as by the US Army Research Laboratory and US Army Research Office grant W911NF-15-1-0233. In addition, the effort of L.H. Nguyen was partially supported by research funds FRG 111172 provided by University of North Carolina at Charlotte. The authors are grateful to Professor Vasilii Astratov from Center for Optoelectronics and Optical Communications of University of North Carolina at Charlotte for many fruitful discussions. 


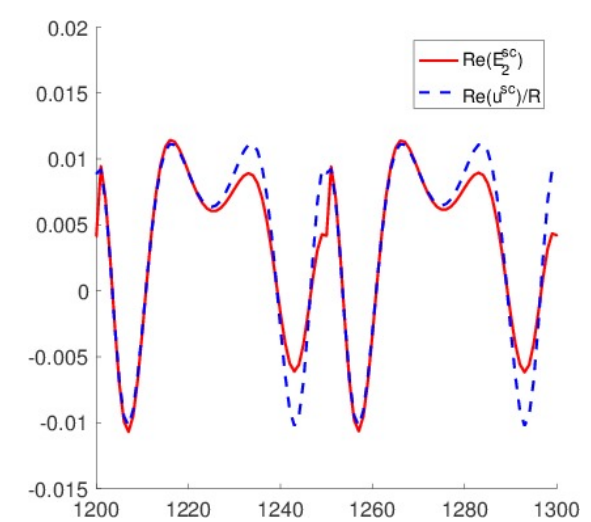

(a)

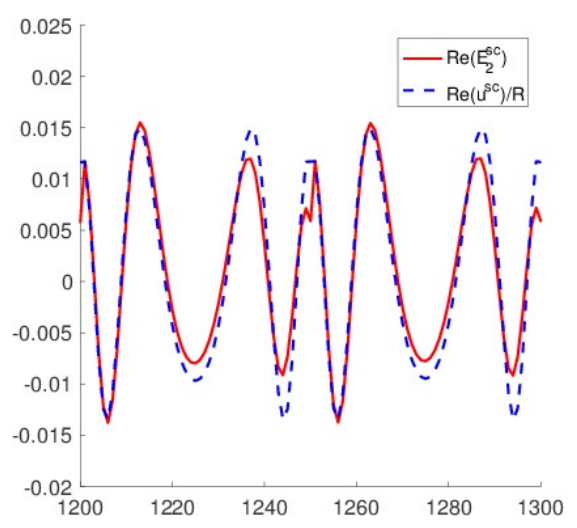

(c)

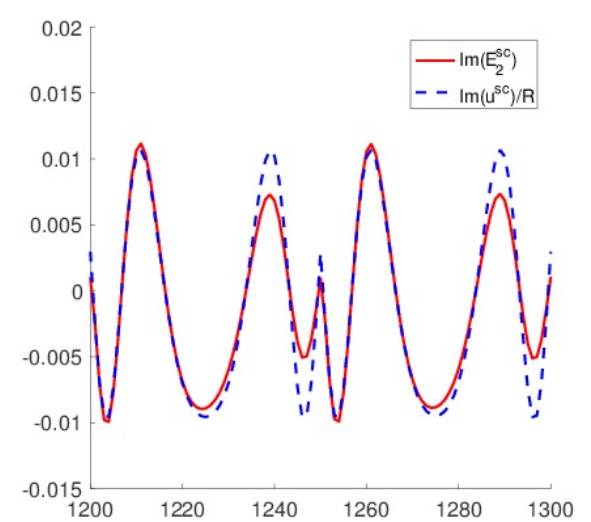

(b)

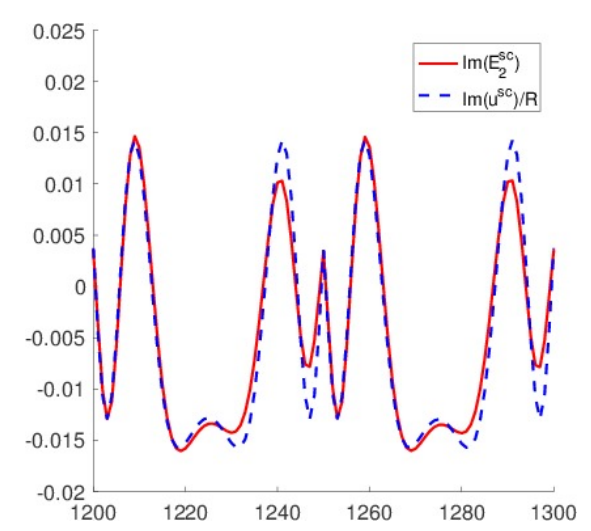

(d)

Figure 7: The real and imaginary parts of $E_{2}^{\mathrm{sc}}$ and $u_{\mathrm{sc}} / R$ at the measurement points from 1200 to 1300. The scattering object is a sphere characterized by a smoothly decaying function. (a) and (b) are for $k=6.5,(c)$ and (d) are for $k=7.5$.

\section{References}

[1] Aktosun T and Sacks P 1998 Inverse problem on the line without phase information Inverse Problems, 14 211-224.

[2] Ammari H, Chow Y, and Zou J. 2016 Phased and phaseless domain reconstruction in inverse scattering problem via scattering coefficients SIAM J. Appl. Math. 76 $1000-1030$.

[3] Ammari H, Garnier J, Jing W, Kang H, Lim M, Solna K, and Wang H 2013 Mathematical and Statistical Methods for Multistatic Imaging, vol. 2098 of Lecture Notes in Mathematics Cham: Springer.

[4] Ammari H and Kang H 2004 Reconstruction of Small Inhomogeneities From Boundary Measurements, vol. 1846 of Lecture Notes in Mathematics, Berlin: Springer.

[5] Bao G and Zhang L 2016 Shape reconstruction of the multi-scale rough surface from multifrequency phaseless data Inverse Problems 32085002

[6] Bardsley P and Guevara Vasquez F 2016 Imaging with power controlled source pairs, SIAM J. Imaging Sci. 9 185-211. 


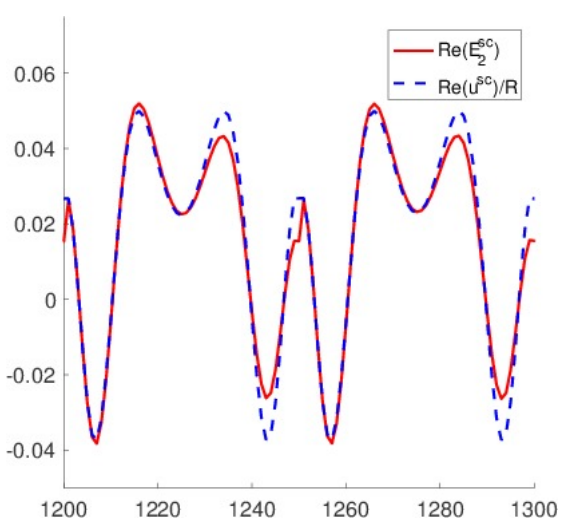

(a)

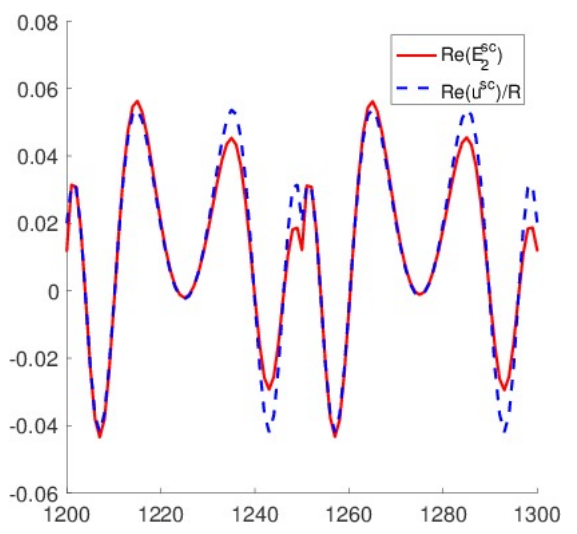

(c)

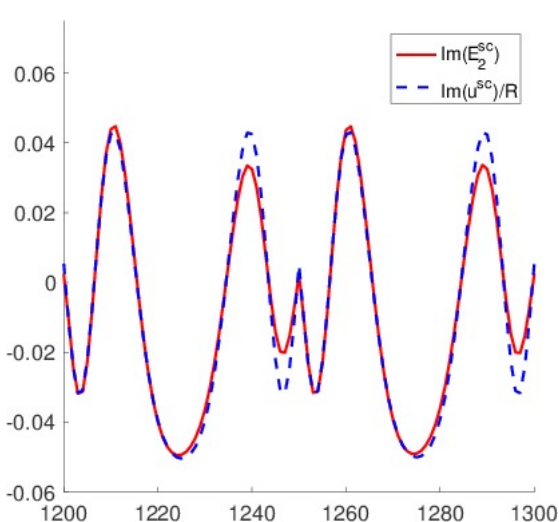

(b)

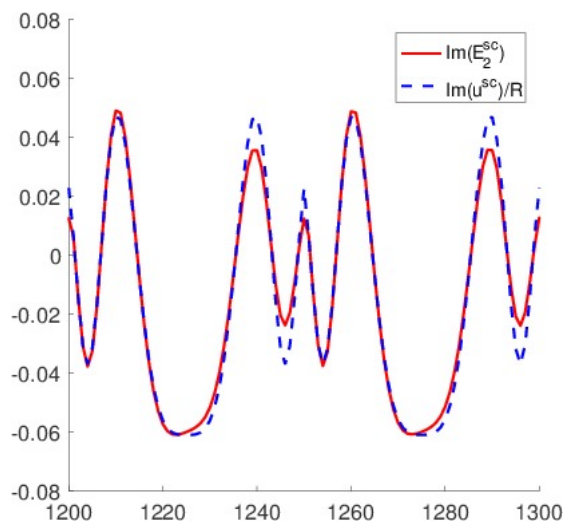

(d)

Figure 8: The real and imaginary parts of $E_{2}^{\mathrm{sc}}$ and $u_{\mathrm{sc}} / R$ at the measurement points from 1200 to 1300. The scattering object is a cube. The coefficient $c(\mathbf{x})$ equals 4.5 inside the scatterer and one elsewhere. (a) and (b) are for $k=6.5,(c)$ and (d) are for $k=7.5$.

[7] Bardsley P and Guevara Vasquez F 2016 Kirchhoff migration without phases, Inverse Problems, 32105006.

[8] Beilina L and Klibanov M V 2012 Approximate Global Convergence and Adaptivity for Coefficient Inverse Problems, Springer, New York.

[9] Bukhgeim A L and Klibanov M V 1981, Uniqueness in the large of a class of multidimensional inverse problems Soviet Math. Doklady 17 244-247.

[10] Chadan K and Sabatier P 1977, Inverse Problems in Quantum Scattering Theory, Springer-Verlag, New York.

[11] Colton D and Kress R 2013 Inverse Acoustic and Electromagnetic Scattering Theory, 3rd edition Springer, New York.

[12] Darahanau A, Nikulin A, Souvorov A, Nishino Y, Muddle B, and Ishikawa T 2005, Nano-resolution profiling of micro-structures using quantitative x-ray phase retrieval from Fraunhofer diffraction data Physics Letters A 335 494-498. 


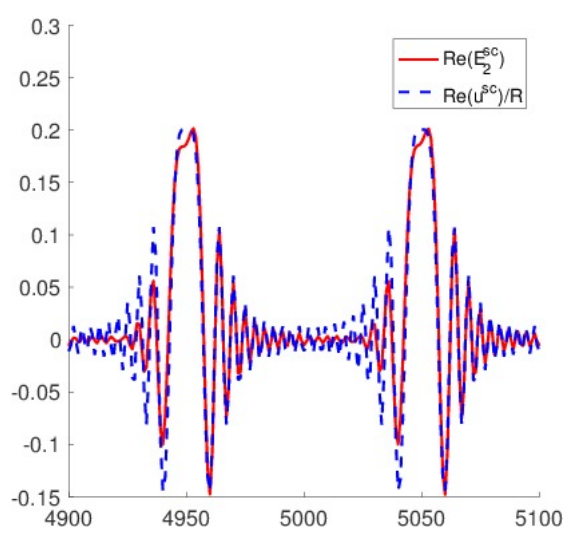

(a) One smooth inclusions, $\mathrm{k}=20.5$

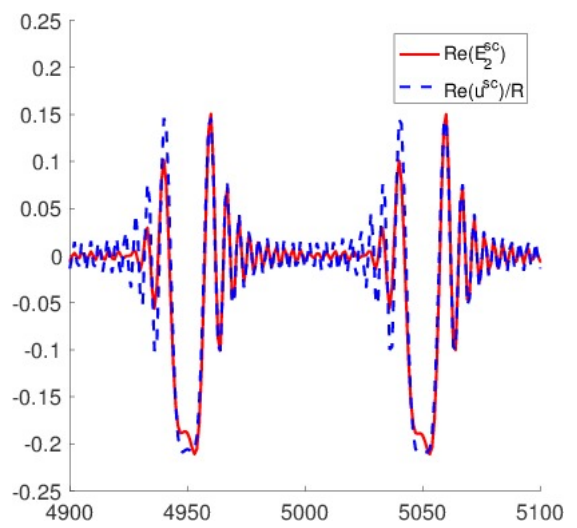

(c) One smooth inclusions, $\mathrm{k}=21.5$

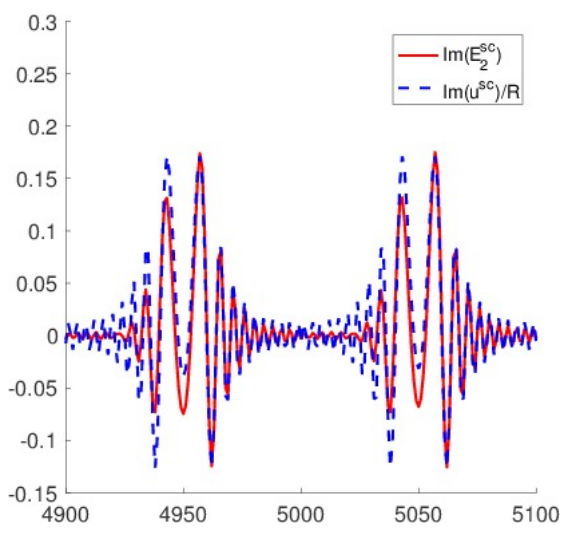

(b) One smooth inclusions, $\mathrm{k}=20.5$

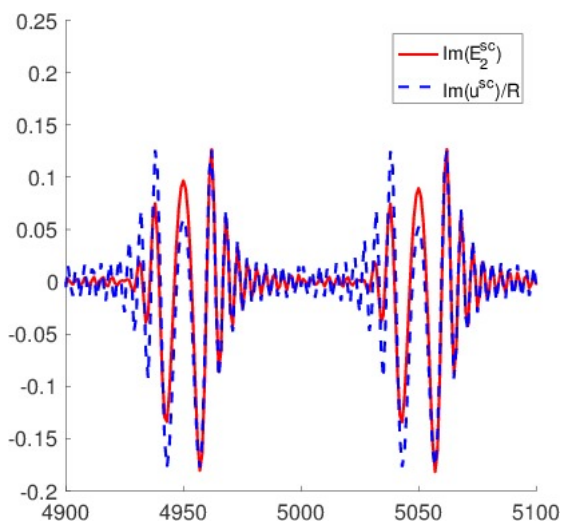

(d) One smooth inclusions, $\mathrm{k}=21.5$

Figure 9: The real and imaginary parts of $E_{2}^{\mathrm{sc}}$ and $u_{\mathrm{sc}} / R$ at the measurement points from 4900 to 5100. The model for the scattering object, which is a sphere, is given in the case of one inclusion in Section 6.4. (a) and (b) are for $k=20.5$, (c) and (d) are for $k=21.5$.

[13] Dierolf M, Bank O, Kynde S, Thibault P, Johnson I, Menzel A, Jefimovs K, David C, Marti O, and Pfeiffer F 2008 Ptychography \& lenseless x-ray imaging, Europhysics News 39 22-24.

[14] Goncharsky A V and Romanov S Y 2017 Iterative methods for solving coefficient inverse problems of wave tomography in models with attenuation Inverse Problems, 33025003.

[15] Ivanyshyn O, Kress R and Serranho P 2010 Huygens' principle, iterative methods in inverse obstacle scattering Adv. Comput. Math. 33 413-429

[16] Ivanyshyn O and Kress R 2011 Inverse scattering for surface impedance from phaseless far field data J. Comput. Phys. 230 3443-52

[17] Khachaturov R 2009 Direct and inverse problems of determining the parameters of multilayer nanostructures from the angular spectrum of the intensity of reflected x-rays Comput. Math. Math. Phys. 49 1781-1788.

[18] Klibanov M V and Timonov A 2004 Carleman Estimates for Coefficient Inverse Problems and Numerical Applications, VSP, Utrecht. 


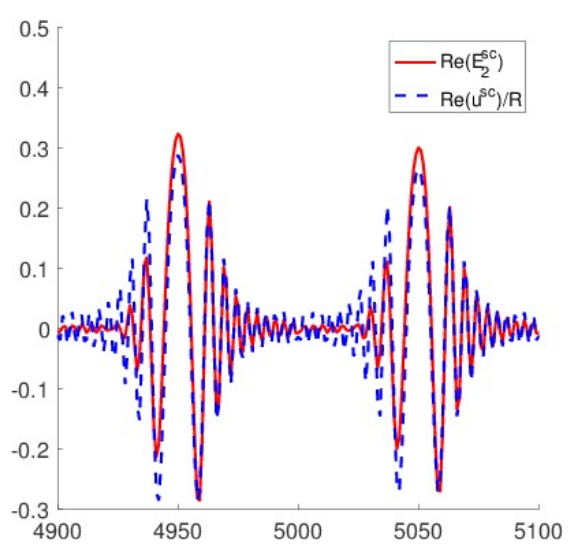

(a) Two smooth inclusions, $\mathrm{k}=20.5$

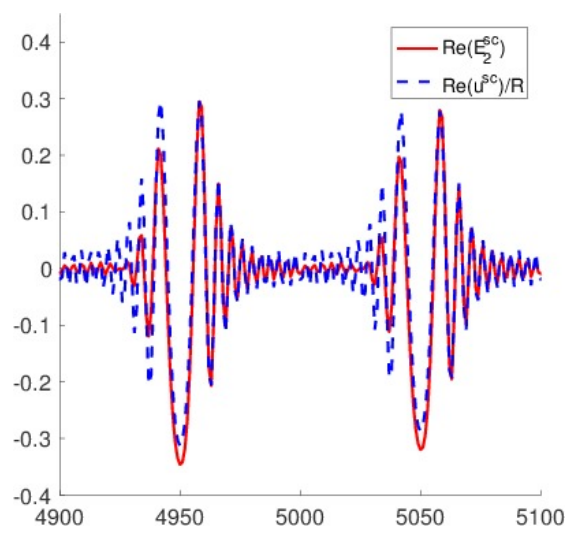

(c) Two smooth inclusions, $\mathrm{k}=21.5$

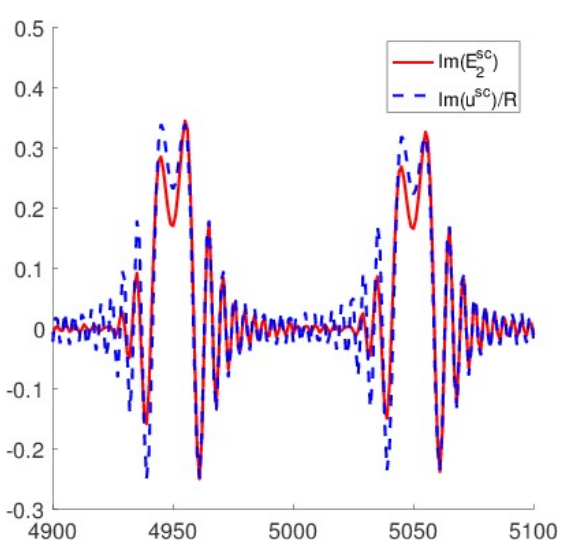

(b) Two smooth inclusions, $\mathrm{k}=20.5$

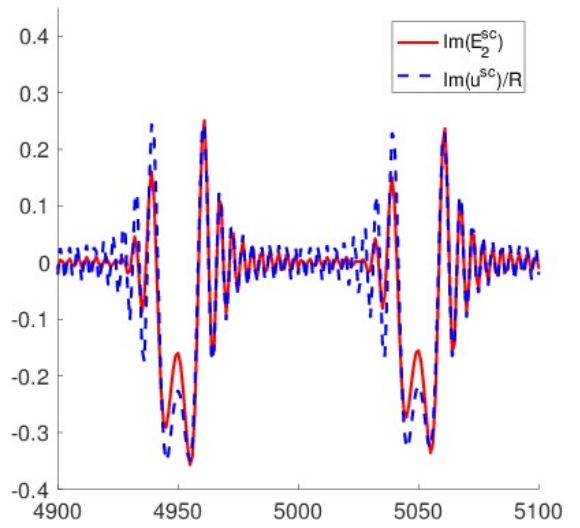

(d) Two smooth inclusions, $\mathrm{k}=21.5$

Figure 10: The real and imaginary parts of $E_{2}^{\mathrm{sc}}$ and $u_{\mathrm{sc}} / R$ at the measurement points from 4900 to 5100. The model for the scattering object, which is two spheres, is given in the case of two inclusions in Section 6.4. (a) and (b) are for $k=20.5$, (c) and (d) are for $k=21.5$.

[19] Klibanov M V 2013, Carleman estimates for global uniqueness, stability and numerical methods for coefficient inverse problems J. Inverse Ill-Posed Probl. 21 477-560.

[20] Klibanov M V, Nguyen L H, and Pan K 2016 Nanostructures imaging via numerical solution of a $3-\mathrm{D}$ inverse scattering problem without the phase information Appl. Numer. Math., 110 190-203.

[21] Klibanov M V and Sacks P 1992 Phaseless inverse scattering and the phase problem in optics J. Math. Phys. 33 3813-3821.

[22] Klibanov M V 2014 Phaseless inverse scattering problems in three dimensions SIAM J. Appl. Math. 74 392-410.

[23] Klibanov M V 2014 On the first solution of a long standing problem: Uniqueness of the phaseless quantum inverse scattering problem in 3-D Appl. Math. Lett. 37 82-85.

[24] Klibanov M V 2014 Uniqueness of two phaseless non-overdetermined inverse acoustics problems in 3-D Appl. Anal. 93 1135-1149. 
[25] Klibanov M V 2017 A phaseless inverse scattering problem for the 3-D Helmholtz equation Inverse Probl. Imaging 11 263-276.

[26] Klibanov M V and Romanov V G 2015 Explicit formula for the solution of the phaseless inverse scattering problem of imaging of nano structures $\mathrm{J}$. Inverse $\mathrm{Ill}$ Posed Probl. 23 187-193.

[27] Klibanov M V and Romanov V G 2015, Explicit solution of 3-D inverse scattering problem for the Schrödinger equation: the plane wave case, Eurasian J. Math. Comput. Appl., 3 48-63.

[28] Klibanov M V and Thành N T 2015 Recovering of dielectric constants of explosives via a globally strictly convex cost functional SIAM J. Appl. Math., 75 518-537.

[29] Klibanov M V and Romanov V G 2016 Reconstruction procedures for two inverse scattering problems without the phase information SIAM J. Appl. Math. 76 178-196.

[30] Klibanov M V and Romanov V G 2016, Two reconsrtuction procedures for a 3-d phaseless inverse scattering problem for the generalized Helmholtz equation, Inverse Problems 32015005.

[31] Klibanov M V and Romanov V G 2017 Uniqueness of a 3-D coefficient inverse scattering problem without the phase information Inverse Problems 33095007.

[32] Klibanov M V, Nguyen D-L, Nguyen L H, and Liu H 2017, A globally convergent numerical method for a 3D coefficient inverse problem with a single measurement of multi-frequency data, Inverse Probl. Imaging, to appear; also see Arxiv 1612.0401.

[33] Kolesov A E, Klibanov M V, Nguyen L H, Nguyen D-L, and Thành N T 2017 Single measurement experimental data for an inverse medium problem inverted by a multifrequency globally convergent numerical method Appl. Num. Math. 120 176-196.

[34] Klibanov M V, Kolesov A E, Nguyen L and A. Sullivan A 2017 Globally strictly convex cost functional for a 1-D inverse medium scattering problem with experimental data SIAM J. Appl. Math. 77, 1733-1755.

[35] Lechleiter A and Nguyen D-L 2014 A trigonometric Galerkin method for volume integral equations arising in TM grating scattering Adv. Comput. Math. 40 1-25.

[36] Li J, Li P, Liu H and Liu X 2015, Recovering multiscale buried anomalies in a twolayered medium, Inverse Problems, 31105006.

[37] Li J, Liu H and Wang Q 2014 Enhanced multilevel linear sampling methods for inverse scattering problems J. Comput. Phys. 257 554-571.

[38] Li J, Liu H and Wang Y 2017 Recovering an electromagnetic obstacle by a few phaseless backscattering measurements Inverse Problems 33, 035011.

[39] Nguyen D-L 2015, A volume integral equation method for periodic scattering problems for anisotropic Maxwell's equations Appl. Numer. Math. 98 59-78. 
[40] Nguyen D-L, Klibanov M V, Nguyen L H, and Fiddy M A 2017 Imaging of buried objects from multi-frequency experimental data using a globally convergent inversion method J. Inverse Ill-Posed Probl., to appear, published online at DOI: 10.1515/jiip2017-0047.

[41] Nguyen D-L, Klibanov M V, Nguyen L H, Kolesov A E, Fiddy M A, and Liu H 2017 Numerical solution of a coefficient inverse problem with multi-frequency experimental raw data by a globally convergent algorithm J. Comput. Phys., 345 17-32.

[42] Novikov R G 1998 A multidimensional inverse spectral problem for the equation $-\Delta \psi+(v(x)-E u(x)) \psi=0$, Funct. Anal. Appl., 22 263-272.

[43] Novikov R G 1992 The inverse scattering problem on a fixed energy level for the two-dimensional Schrödinger operator, J. Funct. Anal., 103 409-463.

[44] Novikov R G 2015 Formulas for phase recovering from phaseless scattering data at fixed frequency Bull. Sci. Math. 139 923-936.

[45] Novikov R G 2016 Explicit formulas and global uniqueness for phaseless inverse scattering in multidimensions, J. Geom. Anal. 26 346-359.

[46] Novikov R G 2015 Phaseless inverse scattering in the one-dimensional case, Eurasian J. Math. Comput. Appl. 3 64-70.

[47] Novotny L and Hecht B 2012, Principles of Nano-Optics, 2nd ed., Cambridge University Press, Cambridge, UK.

[48] Petersena T, Keastb V, and Paganinc D 2008 Quantitative TEM-based phase retrieval of mgo nano-cubes using the transport of intensitive equation Ultramisroscopy 108 805-815.

[49] Phillips R and Milo R 2009, A feeling for numbers in biology, Proc. Natl. Acad. Sci. USA $10621465-71$.

[50] http://kirschner.med.harvard.edu/files/bionumbers/fundamentalBioNumbersHandout.pdf

[51] Romanov V G 1986 Inverse Problems of Mathematical Physics Utrecht: VSP.

[52] Romanov V G 2002 Investigation Methods for Inverse Problems Utrecht: VSP.

[53] Romanov V G 2014 Inverse problems for differential equations with memory Eurasian J. Math. Comput. Appl. 2 51-80

[54] Ruhlandt A, Krenkel M, Bartels M, and Salditt T 2014 Three-dimensional phase retrieval in propagation-based phase-contrast imaging Physical Review A 89033847.

[55] Vainberg B R 1966 Principles of radiation, limiting absorption and limiting amplitude in the general theory of partial differential equations, Russian Math. Surveys 21 115-193.

[56] Vainberg B R 1989 Asymptotic Methods in Equations of Mathematical Physics, Gordon and Breach Science Publishers, New York. 
[57] Xu X, Zhang B and Zhang H 2017 Uniqueness in inverse scattering problems with phaseless far-field data at a fixed frequency, preprint, Arxiv: 1709.07878.

[58] Zhang B and Zhang H 2017 Recovering scattering obstacles by multi-frequency phaseless far-field data, J. Comput. Phys 345 58-73. 